Proceedings of the

International Geometry Center

Vol. 11, no. 4 (2018) pp. 35-71

\title{
On the integrability problem for systems of partial differential equations in one unknown function, I
}

\author{
Antonio Kumpera
}

\begin{abstract}
We discuss the integration problem for systems of partial differential equations in one unknown function and special attention is given to the first order systems. The Grassmannian contact structures are the basic setting for our discussion and the major part of our considerations inquires on the nature of the Cauchy characteristics in view of obtaining the necessary criteria that assure the existence of solutions. In all the practical applications of partial differential equations, what is mostly needed and what in fact is hardest to obtains are the solutions of the system or, occasionally, some specific solutions. This work is based on four most enlightening Mémoires written by Élie Cartan in the beginning of the last century.
\end{abstract}

Анотація. В статті вивчається проблема інтегровності систем $\mathcal{S}$ диференціальних рівнянь в частинних похідних від однієї невідомої функції, причому особливу увагу приділено системам першого порядку. 3 геометричної точки зору ми маємо систему Пфаффа так званої канонічної контактної структури, яка визначається грасмановим пучком контактних елементів певного порядку. Наш підхід є двояким: з одного боку ми використовуємо характеристики Картана для зведення проблеми до мінімальної кількості змінних, а з іншого - досліджуємо характер характеристик Коші для отримання необхідних критеріїв існування розв'язків для системи Пфаффа $\mathcal{P}=\mathcal{P}(\mathcal{S})$, асоційованої з заданою системою рівнянь в частинних похідних $\mathcal{S}$ і отриманої обмеженням вищевказаної канонічної контактної структури на підмноговид $\mathcal{S}$, що визначає дане рівняння. Ми також показуємо, що інтегровність системи $\mathcal{S}$ еквівалентна регулярності характеристик Коші пов'язаних з відповідною розмірністю.

2010 Mathematics Subject Classification: Primary 53C05; Secondary 53C15, 53C17

Keywords: Partial differential equations, Pfaffian system, contact structures, local equivalence.

DOI: http://dx.doi.org/ 
Перша частина статті носить вступний характер і є підготовчою для другої частини в якій вивчатимуться диференціальні рівняння в частинних похідних довільних порядків. Ця робота базується на чотирьох роботах Елі Картана написаних на початку минулого століття (див. бібліографію).

Тим не менш, представлене в ній детальне обговорення характеристик Дарбу, Коші та Картана, виявляе деталі, які, наскільки нам відомо, не були ніде опубліковані. Зокрема, вищезгаданий результат про інтегрування (див. теореми 7.12 і 7.17 ) видається новим кроком у теорії інтегрування диференціальних систем. Також у розділі 5 ми наведено два головних приклади: грасманівський пучок гіперплощин та ліувіллєва структура на кодотичному розшарування, які ілюструють багато ідей описаних в даній роботі.

Зауважимо, що наші методи також застосовуються до випадку неінтегровних систем, і в останніх двох розділах отримано деякі цікаві результати, що ілюструють їх за допомогою систем прапорів. Останні системи можна розглядати як "найбільш неінтегровні" системи Пфаффа не дивлячись на те, що єдиними інтегральними підмноговидами є, власне, інтегральні криві (будь-яка крива, дотична до лінійних контактних елементів, що зануляються системою Пфаффа).

\section{CONTENTS}

\section{Part I}

1 Introduction.

2 Pfaffian systems, definitions and notations.

3 Darboux characteristics.

4 Cartan characteristics.

5 Classical contact structures.

6 Cauchy characteristics.

7 Contact transformations.

\section{Part II}

1 Introduction.

2 Brackets.

3 Higher order differential equations.

4 The local equivalence problem.

5 The local equivalence of differential systems.

6 Integral contact elements.

7 Integral sub-manifolds.

8 The derived systems.

9 The pseudo-groups of local automorphisms.

10 Pfaffian systems whose characters are equal to one.

11 The Cartan co-variant approach.

12 Integration of Pfaffian systems with character equal to one. 
13 Pfaffian systems whose characters are larger than one.

14 Singular Pfaffian Systems.

15 Examples.

\section{INTRODUCTION}

It is our purpose, in this paper, to study the integrability problem for systems $\mathcal{S}$ of partial differential equations in one unknown function, special emphasis being given to first order systems. The geometrical setting is provided by a Pfaffian system, the so-called canonical contact structure, defined on a Grassmann bundle of contact elements of a certain order, our approach being twofold.

Firstly, we make appeal to the Cartan characteristics in view of reducing the problem to the minimum number of variables and, secondly, we inquire on the nature of the Cauchy characteristics in view of obtaining the necessary criteria that will assure the existence of solutions for the Pfaffian system $\mathcal{P}=\mathcal{P}(\mathcal{S})$ canonically associated to $\mathcal{S}$. This latter system is obtained by restricting the above mentioned canonical contact structure to the sub-manifold $\mathcal{S}$ that defines the equation. We prove that the integrability of $\mathcal{S}$ is equivalent to the regularity of the Cauchy characteristics allied to the appropriate dimension. Partial differential equations of arbitrary order will be considered in the second part of this paper,

The present first part is essentially a preparatory digression aimed at setting the necessary foundations for the discussion in the part two where the desired new results do appear. Nevertheless, our present detailed discussion of the characteristics brings out details that, to our knowledge, do not appear in print elsewhere and are the underlying ingredients for our later discussion in the second part. More so, the above mentioned result on integrability and stated in the Theorems 7.12 and 7.17 seems a new step in the integration of differential systems.

\section{Pfaffian systems, Definitions And notations}

Let $M$ be a finite dimensional connected differentiable $\left(C^{\infty}\right)$ manifold, $T=T M$ its tangent bundle and $T^{*}=T^{*} M$ the co-tangent bundle. A regular Pfaffian system defined on $M$ is, by definition, a locally trivial vector sub-bundle $\mathcal{P}$ of $T^{*}$. Its annihilator $\Sigma=\mathcal{P}^{\perp}$ is a sub-bundle of $T,(T / \Sigma)^{*} \simeq \mathcal{P}$ and $T^{*} / \mathcal{P} \simeq \Sigma^{*}$. Such a sub-bundle of $T$ is called a regular distribution, occasionally a field of first order contact elements, on the manifold $M$.

Given a vector bundle $E$ (or, for that matter, any bundle), we denote by $\Gamma(E)$ the module of all the global sections and by $\Gamma_{\ell}(E)$ the pre-sheaf 
composed by the local sections. A local automorphism of $\mathcal{P}$ (or of $\Sigma$ ) is a local diffeomorphism $\varphi$ of $M$ satisfying the property $\varphi^{*} \mathcal{P}=\mathcal{P}$, this property being equivalent, by duality, to $\varphi_{*} \Sigma=\Sigma$. The set $A u t(\mathcal{P})$ of all the local automorphisms of $\mathcal{P}$ is a pseudo-group of transformations of order 1 though not always Lie since it might fail to be complete. An infinitesimal automorphism of $\mathcal{P}$ (or $\Sigma$ ) is a local vector field $\xi$ defined on $M$ and generating a local 1-parameter group of local automorphisms. It can be characterized by the condition $\theta(\xi) \Gamma_{\ell}(\mathcal{P}) \subset \Gamma_{\ell}(\mathcal{P})$ or, equivalently, by $\theta(\xi) \Gamma_{\ell}(\Sigma) \subset \Gamma_{\ell}(\Sigma)$, where $\theta(\xi)$ is the Lie derivative along the vector field $\xi$. The set $\mathcal{L}(\mathcal{P})$ of all the infinitesimal automorphisms of $\mathcal{P}$ is a not necessarily complete infinitesimal pseudo-algebra of order 1 . The eventually singular distribution $L$ induced by $\mathcal{L}(\mathcal{P})$ i.e., defined by $L_{x}=\mathcal{L}(\mathcal{P})_{x}, x \in M$, satisfies the integrability criterion of Stefan and Sussmann, its maximal integral manifolds being the connected components of the orbits of $\operatorname{Aut}(\mathcal{P})$. We say that $\mathcal{P}$ is homogeneous when $\operatorname{Aut}(\mathcal{P})$ operates transitively and infinitesimally homogeneous when $\mathcal{L}(\mathcal{P})$ is transitive i.e., when $L=T M$. Since $M$ is assumed to be connected, infinitesimal homogeneity implies homogeneity, the converse statement being however inexact.

Finally, given a second Paffian system $\mathcal{P}^{\prime}$ on a manifold $M^{\prime}$, we shall say that $(\mathcal{P}, x)$ is locally equivalent to $\left(\mathcal{P}^{\prime}, x^{\prime}\right)$ when there exists a local diffeomorphism $\varphi: U \rightarrow U^{\prime}, U$ and $U^{\prime}$ being open neighborhoods of $x$ and $x^{\prime}$ respectively, such that $\varphi(x)=x^{\prime}$ and $\varphi^{*} \mathcal{P}^{\prime}=\mathcal{P}$. Two systems $\mathcal{P}$ and $\mathcal{P}^{\prime}$ are said to be locally equivalent when the above mentioned property holds for any pair of points $\left(x, x^{\prime}\right) \in M \times M^{\prime}$.

Occasionally, we shall consider distributions and Pfaffian systems that are not regular in the sense adopted in the beginning of this section and shall specify, in each case, the appropriate conditions.

\section{Darboux Characteristics}

In this section we only consider scalar differential forms defined on the manifold $M$ though some of the considerations apply as well to vector valued forms.

Let $\omega$ be an exterior differential form defined on an open subset $U$ of $M$ and denote by $\omega_{x}$ the induced form on $T_{x} M$. The annihilator of $\omega$ at the point $x \in U$ is, by definition, the vector subspace $V_{x} \subset T_{x} M$ defined by

$$
V_{x}=\left\{v \in T_{x} M \mid i(v) \omega_{x}=0\right\} .
$$

When $\omega$ is a 1 -form then $V_{x}=\operatorname{ker} \omega_{x}$. It is easy to prove that $\omega_{x}$ factors to an exterior form defined on $T_{x} / V_{x}$ (i.e., $\omega_{x}=q^{*} \tilde{\omega}_{x}$ where $q$ is the quotient map) and that $V_{x}$ is the largest subspace of $T_{x} M$ having this property. If 
the degree of $\omega$ is equal to $r$, then $V_{x}^{\perp} \subset T^{*} M$ is generated by the family

$$
\left\{i\left(e_{j_{1}} \wedge \cdots \wedge e_{j_{r-1}}\right) \omega\right\}
$$

where $\left\{e_{j}\right\}$ is any set of generators for $T_{x} M$. Moreover, $V_{x}^{\perp}$ is the smallest sub-space $W$ of $T_{x}^{*} M$ such that $\omega_{x}$ belongs to the sub-algebra of $\wedge T_{x}^{*} M$ generated by $\mathbf{R}+W$.

A local automorphism of the form $\omega$ is a local diffeomorphism $\varphi$ of $M$ such that $\varphi^{*} \omega=\omega$ and an infinitesimal automorphism is a local vector field $\xi$ generating a local 1-parameter group of local automorphisms. It is characterized by the condition $\theta(\xi) \omega=0$ in terms of Lie derivatives. The infinitesimal automorphism $\xi$ is said to be characteristic when $i(\xi) \omega=0$ which means that $\xi_{x} \in V_{x}$ for any point $x$ in the domain of $\omega$. Characteristic infinitesimal automorphisms are therefore defined in terms of the equations

$$
i(\xi) \omega=\theta(\xi) \omega=0
$$

or, equivalently, by

$$
i(\xi) \omega=i(\xi) d \omega=0
$$

in view of the relation $\theta(\xi)=[i(\xi), d]$.

The sum and the constant scalar multiples of infinitesimal automorphisms are infinitesimal automorphisms since $\theta([\xi, \eta])=[\theta(\xi), \theta(\eta)]$ and the bracket of infinitesimal automorphisms is again an infinitesimal automorphism. Furthermore, the formula $i([\xi, \eta])=[i(\xi), \theta(\eta)]$ shows that the bracket of characteristic infinitesimal automorphism remains characteristic and, finally, the formulas $i(f \xi)=f i(\xi)$ and $\theta(f \xi)=f \theta(\xi)+d f \wedge i(\xi)$ show that $f \xi$ is characteristic when $\xi$ is, whatever the function $f$. In sum, the set $\mathcal{L}_{c}(\omega)$ of all the characteristic infinitesimal automorphisms of $\omega$ is closed under addition, scalar multiplication by an arbitrary function and under the Lie bracket.

We denote by $\Delta \subset T M$ the eventually singular distribution on the manifold $M$ (or, rather, on the open set $U$ ) induced by the family $\mathcal{L}_{c}(\omega)$ i.e.,

$$
\Delta_{x}=\left\{\xi_{x} \mid \xi \in \mathcal{L}_{c}(\omega)\right\}
$$

and call it the characteristic distribution of $\omega$. Its annihilator $\Delta^{\perp} \subset T^{*} M$ is the characteristic system of $\omega$. The distribution $\Delta$ satisfies the integrability criterion of Stefan and Sussmann and its maximal integral manifolds are the Cauchy characteristics of $\omega$. In view of the equation (3.1), the elements of $\mathcal{L}_{c}(\omega)$ are the local vector fields $\xi$ on $M$ taking values in $\Delta$. When the dimension of $\Delta_{x}, x \in U$, is constant, we say that $\omega$ has regular characteristics in which case $\Delta$ is a regular and integrable distribution on $M$ (rather on $U$ ) and $\Delta^{\perp}$ is a regular Pfaffian system. The following result enables us to reduce, locally, the form $\omega$ to an expression involving 
only the characteristic variables i.e., the first integrals of $\Delta$. The proof is straightforward.

Proposition 3.1. Let $\omega$ be an exterior differential form of degree $r$ with regular characteristics and let $\left\{y_{1}, \cdots, y_{s}\right\}$ be a fundamental system of independent first integrals of $\Delta$ defined in a neighborhood of a given point $x \in M$. Then $\omega$ has the local expression

$$
\omega=\sum a_{i_{1} \cdots i_{r}}(y) d y^{i_{1}} \wedge \cdots \wedge d y^{i_{r}},
$$

where the sum is extended to all the sequences $1 \leq i_{1}<\cdots<i_{r} \leq s$.

Corollary 3.2. Let $\omega$ be an exterior differential form with regular characteristics and let us assume that its degree is equal to the co-dimension of $\Delta$. Then, for any $x \in M$, there exist s first integrals $\left\{z_{1}, \cdots, z_{s}\right\}$ of $\Delta$, defined in a neighborhood of $x$ and such that $\omega=d z_{1} \wedge \cdots \wedge d z_{s}$. These forms are therefore closed and locally decomposable.

Based on the equation (3.1), we now examine a point-wise approach to the characteristics and define, for any $x \in U$, the vector sub-space of $T_{x} M$

$$
\tilde{\Delta}_{x}=\left\{v \in T_{x} M \mid i(v) \omega_{x}=i(v) d \omega_{x}=0\right\} .
$$

In general, $\tilde{\Delta}=\cup \tilde{\Delta}_{x}$ is a singular vector sub-bundle of $T M$ since the dimensions of the fibres can vary and clearly $\Delta \subset \tilde{\Delta}$. When $\omega$ has degree $r$, then $\tilde{\Delta}$ is defined by the linear equations

$$
\left[i\left(w_{1} \wedge \cdots \wedge w_{r-1}\right) \omega\right](v)=\left[i\left(w_{1} \wedge \cdots \wedge w_{r}\right) d \omega\right](v)=0,
$$

where $\left(w_{1}, \cdots, w_{r}\right)$ runs through all the $r$-tuples of vectors in $T_{x} M$ and therefore its annihilator $\tilde{\Delta}^{\perp} \subset T^{*} M$ is generated by the linear forms

$$
\left\{i\left(w_{1} \wedge \cdots \wedge w_{r-1}\right) \omega_{x}, i\left(w_{1} \wedge \cdots \wedge w_{r}\right) d \omega_{x}\right\} .
$$

It suffices, of course, to consider $r$-tuples $\left(e_{i_{1}}, \cdots, e_{i_{r}}\right)$, where $\left\{e_{i}\right\}$ is a basis of $T_{x} M$, such that $1 \leq i_{1}<\cdots<i_{r} \leq \operatorname{dim} M$. Since $\operatorname{dim} \Delta_{x}$ is lower semi-continuous and $\operatorname{dim} \tilde{\Delta}_{x}$ upper semi-continuous, it follows that $\operatorname{dim} \tilde{\Delta}_{x}, x \in U$, is constant if and only if $\Delta=\tilde{\Delta}$. Furthermore, since the dimensions are integers and in the present case bounded, they are locally constant on open dense subsets of $U$ where after the equality of $\Delta_{x}$ with $\tilde{\Delta}_{x}$ also takes place on an open dense subset. The integer $c_{x}=\operatorname{codim} \tilde{\Delta}_{x}$ is precisely the class of $\omega$ at the point $x$. When $\Delta=\tilde{\Delta}$, then $c_{x}$ is constant (assuming $U$ connected), this integer being the Cauchy class of the form $\omega$. In what follows, we state the main results involving the notion of class.

Proposition 3.3. The class of a differential 1 -form $\omega$ is equal to $2 p+1$, at the point $x$, if and only if $\omega_{x} \wedge\left(d \omega_{x}\right)^{p} \neq 0$ and $\left(d \omega_{x}\right)^{p+1}=0$. Under 
these conditions,

$$
\tilde{\Delta}_{x}\left(\omega \wedge d \omega^{\ell}\right)=V_{x}(\omega) \cap V_{x}(d \omega)
$$

for any $\ell=0, \ldots, p$.

Proposition 3.4. The class of a differential 1-form $\omega$ is equal to $2 p$, at the point $x$, if and only if $\left(d \omega_{x}\right)^{p} \neq 0$ and $\omega_{x} \wedge\left(d \omega_{x}\right)^{p}=\left(d \omega_{x}\right)^{p+1}=0$. This being the case,

$$
\tilde{\Delta}_{x}\left(\omega \wedge d \omega^{\ell}\right)=\tilde{\Delta}_{x}\left(d \omega^{q}\right)=V_{x}(d \omega)
$$

for any $\ell=0, \cdots, p-1$ and $q=1, \cdots, p$. If, moreover, $\omega_{x} \neq 0$ then the last condition $\left(d \omega_{x}\right)^{p+1}=0$ is a consequence of the first two.

Proposition 3.5. The class of a closed differential 2-form $\omega$ is, at every point, an even integer. This class is equal to $2 p$, at the point $x$, if and only if $\omega_{x}^{p} \neq 0$ and $\omega_{x}^{p+1}=0$. Under these conditions,

$$
\tilde{\Delta}_{x}\left(\omega^{\ell}\right)=V_{x}(\omega)
$$

for any $\ell=1, \cdots, p$ and $\omega_{x}^{p}$ is decomposable.

Theorem 3.6 (Darboux). Let $\omega$ be a differential 1-form of constant class defined on the manifold $M$. When this class is equal to $2 p+1$ then, at any point $x \in M$, there exist local coordinates $\left(x^{i}\right)$ vanishing at $x$ and such that $\omega$ has the local expression

$$
\omega=d x^{1}+x^{2} d x^{3}+\cdots+x^{2 p} d x^{2 p+1} .
$$

When this class is equal to $2 p$ and, moreover, when $\omega$ is everywhere nonsingular, then it admits the local expression

$$
\omega=\left(1+x^{1}\right) d x^{2}+x^{3} d x^{4}+\cdots+x^{2 p-1} d x^{2 p} .
$$

Theorem 3.7 (Darboux). Let $\omega$ be a closed differential 2-form of constant class equal to $2 p$ defined on the manifold $M$. Then, at any point $x \in M$, there exist local coordinates $\left(x^{i}\right)$, vanishing at $x$, such that $\omega$ has the local expression

$$
\omega=d x^{1} \wedge d x^{2}+d x^{3} \wedge d x^{4}+\cdot+d x^{2 p-1} \wedge d x^{2 p} .
$$

We finally observe, as a consequence of the Corollary 3.2, that nonsingular $n$-forms $(n=\operatorname{dim} M)$ always have the local expression

$$
\omega=d x^{1} \wedge \cdots \wedge d x^{n}
$$

non-singular closed $(n-1)$-forms the local expression

$$
\omega=d x^{1} \wedge \cdots \wedge d x^{n-1}
$$


and non-singular $(n-1)$-forms satisfying $d \omega_{x} \neq 0$ the local expression

$$
\omega=\left(1+x^{1}\right) d x^{2} \wedge \cdots \wedge d x^{n}
$$

in a neighborhood of $x$. In all the above displayed expressions of the local canonical forms, the coordinates $x^{i}$ figuring in these expressions are necessarily first integrals of the characteristic distribution $\Delta$ namely, they are the so-called characteristic functions.

\section{Cartan characteristics}

Let $\mathcal{P}$ be a Pfaffian system defined on the manifold $M$. Inasmuch as above, an infinitesimal automorphism $\xi \in \mathcal{L}(\mathcal{P})$ is said to be characteristic whenever it is tangent to $\Sigma$ i.e., when $\xi \in \Gamma_{\ell}(\Sigma)$. The set $\mathcal{L}_{c}(\mathcal{P})=\mathcal{L}(\mathcal{P}) \cap \Gamma_{\ell}(\Sigma)$ of all the characteristic vector fields of the system is an infinitesimal pseudo-algebra of order 1. Moreover, it is a pre-sheaf of modules with respect to the local $C^{\infty}$-functions on $M$ hence $f \xi$ is characteristic whenever $\xi$ is, $f$ being any local function on $M$. We remark that Frobenius' integrability condition, in terms of brackets, amounts to say that $\mathcal{P}$ is integrable if and only if $\mathcal{L}_{c}(\mathcal{P})=\Gamma_{\ell}(\Sigma)$. The module $\mathcal{L}_{c}(\mathcal{P})$ induces an eventually singular integrable distribution $\Delta: x \in M \rightarrow \Delta_{x}=\mathcal{L}_{c}(\mathcal{P})_{x}$, called the characteristic distribution of $\mathcal{P}$, its annihilator $\Delta^{\perp} \subset T^{*} M$ being the characteristic system of $\mathcal{P}$. The maximal integral manifolds of $\Delta$ are the Cartan characteristics of $\mathcal{P}$. Again, $\mathcal{P}$ is integrable if and only if $\Delta=\Sigma$, in which case the Cartan characteristics coincide with the integral leaves of $\Sigma$. Since $\theta(\xi)=i(\xi) d+d i(\xi)$, it follows that $\mathcal{L}_{c}(\mathcal{P})$ is the pre-sheaf of all the local vector fields $\xi$ on $M$ satisfying the equations

$$
\langle\xi, \omega\rangle=\langle\xi, i(\eta) d \omega\rangle
$$

for all $\omega \in \Gamma_{\ell}(\mathcal{P})$ and $\eta \in \Gamma_{\ell}(\Sigma)$, and therefore $\mathcal{L}_{c}(\mathcal{P})$ is the set of all the local vector fields on $M$ taking values in $\Delta$. When the dimension of $\Delta_{x}, x \in$ $M$, is locally constant, we shall say that $\mathcal{P}$ has regular characteristics. The result that follows provides a method enabling us to reduce locally the Pfaffian system $\mathcal{P}$ to the sole characteristic variables i.e., to the first integrals of $\Delta$.

Theorem 4.1 (Cartan). Let $\mathcal{P}$ be a Pfaffian system with regular characteristics and $\left\{y^{1}, \cdots, y^{s}\right\}$ a fundamental system of independent first integrals of $\Delta$ defined in a neighborhood of a given point $x \in M$. There exists then a local basis $\left\{\omega^{1}, \cdots, \omega^{r}\right\}$ of $\mathcal{P}$, defined in a neighborhood of $x$, such that

$$
\omega^{i}=\sum a_{j}^{i}\left(y^{1}, \cdots, y^{s}\right) d y^{j}, 1 \leq i \leq r .
$$

Given a regular integrable distribution $\Sigma$ on the manifold $M$, we say that the open set $U$ is simple with respect to $\Sigma$ when the foliation associated to 
the restricted distribution $\Sigma \mid U$ admits a quotient, this meaning precisely that the quotient space $U / \Sigma$, modulo the leaves of $\Sigma \mid U$, admits a manifold structure for which the quotient map $\rho: U \rightarrow U / \Sigma$ is a surmersion. The manifold structure of $U / \Sigma$ is then uniquely determined by the structure of $U$.

Expressed in geometrical terms, the previous theorem can be transcribed as follows enhancing the role of the finite automorphisms.

Corollary 4.2. Let $\mathcal{P}$ be a Pfaffian system with regular characteristics, $U$ an open set of $M$ simple with respect to the characteristic distribution $\Delta$ and $\rho: U \rightarrow U / \Delta$ the quotient surmersion modulo the leaves of $\Delta$. Then there exists a unique Pfaffian system $\tilde{\mathcal{P}}$, defined on $U / \Delta$, such that

$$
\rho^{*} \tilde{\mathcal{P}}=\mathcal{P} \mid U
$$

Moreover, $\mathcal{L}_{c}(\tilde{\mathcal{P}})=0$, the finite and infinitesimal automorphisms of $\mathcal{P} \mid U$ are locally $\rho$-projectable onto the corresponding automorphisms of $\tilde{\mathcal{P}}$ (they are globally projectable when their domains have connected $\rho$-fibres) and, for any $x \in U$, there exists an open neighborhood $U_{x}$ such that the projections $\mathcal{L}\left(\mathcal{P} \mid U_{x}\right) \rightarrow \mathcal{L}\left(\tilde{\mathcal{P}} \mid \rho\left(U_{x}\right)\right)$ and $\operatorname{Aut}\left(\mathcal{P} \mid U_{x}\right) \rightarrow \operatorname{Aut}\left(\tilde{\mathcal{P}} \mid \rho\left(U_{x}\right)\right)$ are surjective. Finally, any integral manifold $\tilde{\mathcal{V}}$ of $\tilde{\mathcal{P}}$ (not necessarily of maximal dimension) gives rise, by a lift up process, to the integral manifold $\rho^{-1}(\tilde{\mathcal{V}})$ of $\mathcal{P}$.

Conversely, the following statement also holds.

Corollary 4.3. Let $\mathcal{P}$ be a Pfaffian system defined on the manifold $M$ and let us assume that, for every $x \in M$, there exists an open neighborhood $U_{x}$, a surmersion $\rho_{x}: U_{x} \rightarrow V_{x}$ onto a manifold $V_{x}$ and a Pfaffian system $\tilde{\mathcal{P}}_{x}$, defined on $V_{x}$, having null characteristics $\left(\right.$ i.e., $\mathcal{L}_{c}\left(\tilde{\mathcal{P}}_{x}\right)=0$ ) and such that $\mathcal{P} \mid U_{x}=\rho_{x}^{*}\left(\tilde{\mathcal{P}}_{x}\right)$. The dimension of $\Delta_{x}$ is then constant (i.e., $\mathcal{P}$ has regular characteristics), each $U_{x}$ is simple with respect to $\Delta$ and the family $\left(\rho_{x}\right)$ is a foliated atlas for the characteristic foliation of $\mathcal{P}$.

With the notations of the Corollary 4.2, we shall say that each quotient manifold $U / \Delta$ is a space of characteristic variables for $\mathcal{P}$ and the system $\tilde{\mathcal{P}}$ will be called a local reduction of $\mathcal{P}$ to its characteristic variables. Furthermore, a complete set of local first integrals of $\Delta$ will be called a complete set, or system, of characteristic variables for $\mathcal{P}$.

It is useful to examine the point-wise approach to the calculation of the characteristics, this being actually the standard approach. Based on the relation (4.1), we define, for every point $x \in M$, the vector sub-space of 
$T_{x} M$,

$$
\tilde{\Delta}_{x}=\left\{v \in \Sigma_{x} \mid i(v) d \omega \in \mathcal{P}_{x}, \forall \omega \in \Gamma_{\ell}(\mathcal{P})\right\} .
$$

Since this space is defined by the linear equations $\langle v, i(w) d \omega\rangle=0$, with $w \in \Sigma_{x}$ and $\omega \in \Gamma_{\ell}(\mathcal{P})$, its annihilator $\tilde{\Delta}_{x}^{\perp}$ is the subspace of $T_{x}^{*} M$ generated by

$$
\left\{\omega_{x}, i(w) d \omega_{x} \mid w \in \Sigma_{x}, \omega \in \Gamma_{\ell}(\mathcal{P})\right\} .
$$

The family $\tilde{\Delta}=\left(\tilde{\Delta}_{x}\right)$ is, in general, a singular vector sub-bundle of $T M$ (the fibres might not have, locally, constant dimensions) even if $\Delta$ is regular. The integer $s_{x}=\operatorname{codim} \tilde{\Delta}_{x}$ is called the class of $\mathcal{P}$ or of $\Sigma$ at the point $x \in M$. We observe that $\Delta \subset \tilde{\Delta}$ and that $\mathcal{L}_{c}(\mathcal{P})$ is the pre-sheaf of all the local vector fields $\xi$ on $M$ taking values in $\tilde{\Delta}$.

Furthermore and on account of a semi-continuity argument, the equality $\Delta=\tilde{\Delta}$ holds locally if and only if either $\Delta$ or $\tilde{\Delta}$ have, locally, constant dimension. When this equality occurs, the integer $s=s_{x}$, whose value is locally independent of the point $x$, is called the class of the Pfaffian system $\mathcal{P}$ or of the distribution $\Sigma$.

Usually, the Darboux and the Cartan Theorems are proved under more restrictive assumptions on the class constancy though this is quite irrelevant. In any case, both distributions $\Delta$ and $\tilde{\Delta}$ have locally a constant dimension in open and everywhere dense subsets of $M$, the subset corresponding to the later being contained in that corresponding to the former. One last remark is due namely, that the preceding corollaries admit the following additional comment. If, in the corollaries, one of the systems $\mathcal{P}$ or $\tilde{\mathcal{P}}$ satisfies $\Delta=\tilde{\Delta}$ the same will also hold for the other.

\section{Classical contact structures}

In this section we consider a distribution $\Sigma$ of co-dimension 1 namely, a field of hyperplanes or, equivalently, a Pfaffian system of rank 1 defined on the manifold $M$, and recall a few basic facts.

Lemma 5.1. Let $\mathcal{P}$ be a Pfaffian system of rank 1 . Then, for any point $x \in M$, the co-dimension of $\tilde{\Delta}_{x}$ is odd and consequently the class of the system $\mathcal{P}$ is always odd.

When $\tilde{\Delta}_{x}=0\left(\right.$ resp. $\left.\operatorname{dim} \tilde{\Delta}_{x}=1\right)$, then forcibly the dimension of $M$ is odd (resp. even). The results that follow establish the relationship between the Darboux class of the local generators $\omega$ and the Cartan class of $\mathcal{P}$.

Proposition 5.2. Let $\mathcal{P}$ be a Pfaffian system of rank 1 defined on the manifold $M$. Then the following properties are equivalent:

(a) The class of $\mathcal{P}$ is maximum (i.e., $\tilde{\Delta}_{x}=0$ ). 
(b) $\operatorname{dim} M=2 n+1$ and any local generator $\omega \in \Gamma_{\ell}(\mathcal{P})-0$ satisfies the condition $\omega \wedge(d \omega)^{n} \neq 0$ everywhere.

(c) $\operatorname{dim} M=2 n+1$ and, for any point $x \in M$, there exists an element $\omega \in \Gamma_{\ell}(\mathcal{P})-0$, defined in a neighborhood of $x$, such that $\omega_{x} \wedge\left(d \omega_{x}\right)^{n} \neq 0$.

Pfaffian systems of rank 1 and maximum class equal to $\operatorname{dim} M$ are, in view of the property (b), the classical contact structures on odd dimensional manifolds defined by a covering $\left(U_{\alpha}, \omega_{\alpha}\right)$ where the $\omega_{\alpha}$ are differential 1forms of maximum Darboux class, defined on the open sets $U_{\alpha}$, and satisfy the compatibility condition $\omega_{\beta}=f_{\beta \alpha} \omega_{\alpha}$ on the overlap $U_{\alpha} \cap U_{\beta}$.

Proposition 5.3. Let $\mathcal{P}$ be a Pfaffian system of rank 1 on the manifold M. Then, the following properties are equivalent:

(a) The class of $\mathcal{P}$ is equal to $\operatorname{dim} M-1$ or, equivalently, $\operatorname{dim} \tilde{\Delta}_{x}=1$.

(b) $\operatorname{dim} M=2 n+2$ and any local generator $\omega \in \Gamma_{\ell}(\mathcal{P}-0)$ satisfies everywhere the condition $\omega \wedge(d \omega)^{n} \neq 0$.

(c) $\operatorname{dim} M=2 n+2$ and, for any $x \in M$, there exists $\omega \in \Gamma_{\ell}(\mathcal{P}-0)$, defined in a neighborhood of $x$, such that $\omega_{x} \wedge\left(d \omega_{x}\right)^{n} \neq 0$.

(d) $\operatorname{dim} M=2 n+2$ and, for any $x \in M$, there exists $\omega \in \Gamma_{\ell}(\mathcal{P}-0)$, defined in a neighborhood of $x$, such that $\left(d \omega_{x}\right)^{n+1} \neq 0$.

(e) $\operatorname{dim} M=2 n+2$ and, for any $x \in M$, there exists $\omega \in \Gamma_{\ell}(\mathcal{P}-0)$, defined in a neighborhood of $x$, such that $\omega_{x} \wedge\left(d \omega_{x}\right)^{n} \neq 0$ and $(d \omega)^{n+1}=0$.

Pfaffian systems of rank 1 and class equal to $\operatorname{dim} M-1$ are, in view of the property $(d)$, the classical contact structures on even dimensional manifolds defined by a covering $\left(U_{\alpha}, \omega_{\alpha}\right)$ where each $\omega_{\alpha}$ is a Pfaffian form of maximum Darboux class defined on the open sets $U_{\alpha}$, these forms satisfying the compatibility condition $\omega_{\beta}=f_{\beta \alpha} \omega_{\alpha}$ on the overlap. Any such structure is, locally, the inverse image of an odd classical contact structure.

We next indicate a general result, consequence of the Cartan Theorem, concerning Pfaffian systems of rank 1 and constant class. Initially, we state the following

Lemma 5.4. Given a Pfaffian system $\mathcal{P}$ on the manifold $M$, the following properties are equivalent:

(a) The rank of $\mathcal{P}$ is equal to 1 and the class is constant (i.e., $\Delta=\tilde{\Delta}$ ).

(b) There exists, for any $x \in M$, an open neighborhood $U$, a submersion $\rho: U \rightarrow \mathcal{U}$ and an odd classical contact structure $\tilde{\mathcal{P}}$, on $\mathcal{U}$, such that $\mathcal{P} \mid U=\rho^{*} \tilde{\mathcal{P}}$. 
When these equivalent conditions are satisfied, the equality

$$
\text { class } \mathcal{P}=\operatorname{class} \tilde{\mathcal{P}}
$$

holds. In particular, the even classical contact structures are precisely those Pfaffian structures obtained, locally, as inverse images via submersions $\rho$ with 1-dimensional fibres.

Theorem 5.5. Let $\mathcal{P}$ be a Pfaffian system of rank 1 on the manifold $M$. Then the following properties are equivalent:

(a) The class of $\mathcal{P}$ is constant and equal to $2 p+1$.

(b) Any $\omega \in \Gamma_{\ell}(\mathcal{P}-0)$ satisfies, everywhere, the conditions

$$
\omega \wedge(d \omega)^{p} \neq 0 \quad \text { and } \quad \omega \wedge(d \omega)^{p+1}=0 .
$$

(c) For any $x \in M$, there exists a form $\omega \in \Gamma_{\ell}(\mathcal{P}-0)$, defined in a neighborhood of $x$, such that

$$
\omega_{x} \wedge\left(d \omega_{x}\right)^{p} \neq 0 \quad \text { and } \quad \omega_{x} \wedge\left(d \omega_{x}\right)^{p+1}=0 .
$$

(d) For any $x \in M$, there exists a form $\omega \in \Gamma_{\ell}(\mathcal{P}-0)$, defined in a neighborhood of $x$, such that

$$
\omega_{x} \wedge\left(d \omega_{x}\right)^{p} \neq 0 \quad \text { and } \quad(d \omega)^{p+1}=0 .
$$

(e) If $\operatorname{dim} M>2 p+1$, then, for any $x \in M$, there exists $\omega \in \Gamma_{\ell}(\mathcal{P}-0)$, defined in a neighborhood of $x$, such that

$$
\left(d \omega_{x}\right)^{p+1} \neq 0 \quad \text { and } \quad \omega_{x} \wedge\left(d \omega_{x}\right)^{p+1}=0 .
$$

Corollary 5.6. Two Pfaffian structures $(\mathcal{P}, M)$ and $\left(\mathcal{P}^{\prime}, M^{\prime}\right)$, of rank 1 and constant class, are locally equivalent if and only if $\operatorname{dim} M=\operatorname{dim} M^{\prime}$ and class $\mathcal{P}=$ class $\mathcal{P}^{\prime}$. In particular, any such Pfaffian structure is transitive.

We shall now illustrate the previous discussion by examining two among the most relevant classical contact structures namely, the Grassmann or canonical contact structure on the manifold of hyperplanes and the Liouville structure on the cotangent bundle.

Example 5.7. The Grassmannian bundle of hyperplanes. Let $M$ be a manifold of dimension equal to $n+1$ and let us denote by $G(M)$ the Grassmannian bundle of all the hyperplanes contained in the various tangent spaces to the manifold $M$.

We indicate the elements of $G(M)$ by $H$ or by $H_{x}$ whenever it is desirable to specify the base point $x \in M\left(H_{x} \subset T_{x} M\right)$ and by $\pi: T M \rightarrow M$ the standard projection. Local coordinates can be assigned to $G(M)$ as follows: Let $H_{a}$ be an element of $G(M)$ and $\left(U, x^{i}\right), 1 \leq i \leq n+1$, any coordinate system on $M$ with domain $U$, containing the point $a$. There 
exists a sequence $1 \leq i_{1}<\cdots<i_{n} \leq n+1$ for which the restricted linear forms $d x^{i_{1}}\left|H_{a}, \cdots, d x^{i_{n}}\right| H_{a}$ constitute a basis of $H_{a}^{*}$. Let us assume for convenience that $i_{j}=j$, denote by $y$ the remaining coordinate $x^{n+1}$ and consider the subset $\mathcal{U} \subset \pi^{-1} U$ of all the hyperplanes $H$ for which the family $\left\{d x^{i} \mid H\right\}, 1 \leq i \leq n$, is free. Coordinates $\left(x^{i}, y, p_{j}\right)$ can now be assigned to any element $H \in \mathcal{U}$ by simply taking the coordinates $\left(x^{i}, y\right)$ of $\pi(H)$ and writing

$$
d y \mid H=\sum p_{i} d x^{i}, \quad 1 \leq i \leq n .
$$

If $N \subset M$ is a sub-manifold of co-dimension 1 and $T_{a} N \in \mathcal{U}$ then, by the inverse function theorem, $N$ is locally, in a neighborhood of $a$, the graph of a function $y=f\left(x^{1}, \cdots, x^{n}\right), p_{i}\left(T_{a} N\right)=\left(\partial f / \partial x^{i}\right)_{b}$, and $b=\left(x^{i}(a)\right)$.

We observe that $G(M)$ can be identified with the real projective bundle $R P\left(T^{*} M\right)$ since any hyperplane $H \subset T_{x} M$ is annihilated by a line in $T_{x}^{*} M$. A simple computation shows that the coordinates $p_{i}$ are, up to a sign, the inhomogeneous projective coordinates in the fibres of $R P\left(T^{*} M\right)$. More precisely, $p_{i}=-\tilde{p}_{i} / \tilde{p}_{n+1}, 1 \leq i \leq n$, where the $\left(\tilde{p}_{1}, \cdots, \tilde{p}_{n+1}\right)$ are homogeneous coordinates on the fibres of $R P\left(T^{*} M\right)$.

We next consider the line bundle $\Phi$ on $G(M)$ whose fibre, at the point $H$, is equal to $T_{\pi(H)} M / H$. This bundle is simply the quotient $\pi^{-1}(T M) / \mathcal{E}$, where $\mathcal{E}$ is the canonical vector bundle on $G(M)$ with fibre $\mathcal{E}_{H}=H$. The fundamental form $\Omega$ on $G(M)$ with values in $\Phi$ can now be defined as the quotient of $T \pi \bmod \mathcal{E}$ namely,

$$
\Omega(v)=T \pi(v) \bmod H,
$$

for any $v \in T_{H} G(M)$. Taking the coordinates $\left(\mathcal{U} ; x^{i}, y, p_{j}\right)$ introduced earlier and observing that the vector field $\partial / \partial y$, on $G(M)$, factors to an everywhere non-vanishing local section $[\partial / \partial y]$ of the bundle $\Phi$, we infer from the relation (5.1) that

$$
\Omega \mid \mathcal{U}=\omega[\partial / \partial y]
$$

where

$$
\omega=d y-\sum p_{i} d x^{i}
$$

Let $\Sigma=\operatorname{ker} \Omega \subset T G(M)$ and $\mathcal{P}=\Sigma^{\perp}$. The Pfaffian system $\mathcal{P}$ is called the canonical contact structure on $G(M)$ and, since it is generated on the open set $\mathcal{U}$ by the contact form $\omega$, we infer from the Proposition 5.2 that $\mathcal{P}$ is an odd classical contact structure. A simple computation of the characteristic system will now show that the class of $\mathcal{P}$ is equal to $2 n+1$. In fact, it suffices to observe that $\Sigma_{H}=T \pi^{-1}(H)$ and thereafter determine a set of free generators for $\widetilde{\Delta}_{H}^{\perp}$. 
It is interesting to observe that $\Sigma_{H}$ is composed by those tangent vectors $v$ to the point $H$ corresponding to the infinitesimal motions tangent to $H$ i.e., the infinitesimal motions of the base point $x$ that are contained in $H$.

Example 5.8. The Liouville structure on the cotangent bundle. Let us now turn our attention to the Liouville structure on $T^{*} M$. For this, we define the distribution $\bar{\Sigma}$ on the cotangent bundle $T^{*} M$ by assigning, to each point $\mu$, the hyperplane $\bar{\Sigma}_{\mu}=\left(T_{\mu} q\right)^{-1}(\operatorname{ker} \mu)$ where $q: T^{*} M \rightarrow M$ is the standard projection.

Denoting by $\lambda$ the Liouville form on $T^{*} M$ defined by $\lambda_{\mu}=q^{*} \mu$ i.e., $\lambda_{\mu}(v)=\left\langle\left(T_{\mu} q\right) v, \mu\right\rangle$, it follows that $\bar{\Sigma}_{\mu}=\operatorname{ker} \lambda_{\mu}$ hence the Pfaffian system $\overline{\mathcal{P}}=\bar{\Sigma}^{\perp}$, defined on the manifold $T^{*} M$, is generated by the Liouville form $\lambda$. The distribution $\bar{\Sigma}$ as well as the Pfaffian system $\overline{\mathcal{P}}$ are regular on $T^{*} M-0$, where 0 stands for the null section, the latter being called the Liouville structure on the co-tangent bundle. Taking local coordinates $\left(x^{i}, p_{i}\right)$, the Liouville form assumes the expression $\lambda=\sum p_{i} d x^{i}$ hence its Darboux class is equal to $2 n+2(\operatorname{dim} M=n+1)$ and consequently, according to the Proposition $5.2, \overline{\mathcal{P}}$ is an even classical contact structure on $T^{*} M$. It is nevertheless interesting to have a closer look at the characteristic system.

A straightforward calculation, using the above specified coordinates, will show that the characteristic subspace $\widetilde{\Delta}_{\mu}$ is contained in the tangent space, at the point $\mu$, to the fibre $T_{x}^{*} M, x=q(\mu)$, and, since we can identify canonically $T_{\mu} T_{x}^{*} M$ with $T_{x}^{*} M$, it also follows that $\widetilde{\Delta}_{\mu}$ is the 1 -dimensional subspace of $T_{\mu} T_{x}^{*} M$ generated by $\mu$. The tangent co-vector $\mu$ belongs therefore to $\widetilde{\Delta}_{\mu}$.

We shall try, next, to retrace the above considerations within a more geometrical context. Let $L$ be the Liouville vector field on $T^{*} M$ namely, the infinitesimal generator of the 1-parameter group of homothetical transformations $(t, \mu) \mapsto h_{t}(u)=e^{t} \mu$. A simple calculation shows, under the above-mentioned identification, that $L$ is the vertical vector field (tangent to the fibres) defined by $L_{\mu}=\mu$ and, therefore, that $L=\sum p_{i} \partial / \partial p_{i}$ in local coordinates. The previous discussion also shows that $L$ generates $\widetilde{\Delta}=\Delta$ on $T^{*} M-0$, a result that follows directly upon using the homothetical transformations. Let us also observe that $\theta(L) \lambda=\lambda$ and, since $\langle L, \lambda\rangle=0$, that the Liouville vector field is characteristic, hence the characteristic algebra of $\overline{\mathcal{P}}$ is precisely equal to the set of all the multiples $\{f L\}$ where $f$ is an arbitrary function.

The maximal integral manifolds of the characteristic distribution $\Delta$ are the rays, emanating from the origin, in the various fibres of $T^{*} M-0$, hence the characteristic foliation admits a quotient manifold $\left(T^{*} M-0\right) / \Delta$ 
that is diffeomorphic to a sphere bundle over $M$. The Pfaffian system $\overline{\mathcal{P}}$ then factors to a system $\widetilde{\mathcal{P}}$ on the quotient manifold, the class of $\widetilde{\mathcal{P}}$ being maximum and equal to $\operatorname{dim}\left(T^{*} M-0\right) / \Delta=2 n+1$. The system $\widetilde{\mathcal{P}}$ is therefore an odd classical contact structure as predicted, locally, by Lemma 5.4.

We can however do slightly better. From the commutativity of the diagram

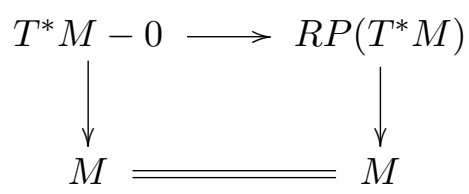

and the sole definitions of $\Sigma$ and $\bar{\Sigma}$, it follows that $\bar{\Sigma}$ projects onto $\Sigma$ via the quotient map though the Liouville form $\lambda$ cannot project onto a generator of $\mathcal{P}$. However, restricting our attention to the open subsets where $p_{n+1} \neq 0$, we can replace the generator $\lambda$ of $\overline{\mathcal{P}}$ by the local generator

$$
\left(1 / p_{n+1}\right) \lambda=d x^{n+1}+\sum\left(p_{i} / p_{n+1}\right) d x^{i},
$$

the later form projecting onto $R P\left(T^{*} M\right)$ and giving rise to the standard generator $\omega=d y-\sum p_{i} d x^{i}$ of $\mathcal{P}$ since $p_{i} / p_{n+1}=-\tilde{p}_{i} / \tilde{p}_{n+1}$ and $x^{n+1}=y$, the right hand side tildet coordinates being those considered in the first example.

The system $\widetilde{\mathcal{P}}$ obtained by reducing $\overline{\mathcal{P}}$ to its characteristic variables sits on the twofold covering space $\left(T^{*} M-0\right) / \Delta$ of $G(M)$. It is rather interesting that $\widetilde{\mathcal{P}}$ can further be reduced to $\mathcal{P}$. As a consequence of the last corollary, we can also state the following

Corollary 5.9. Any odd classical contact structure is locally equivalent to the Grassmann structure on the manifold of hyperplanes and any even structure is locally equivalent to the Liouville structure on a co-tangent bundle.

\section{Cauchy characteristics}

We consider again the canonical contact structure $\mathcal{P}$ defined on the Grassmannian bundle $G(M)$ by the fundamental form $\Omega$, the manifold $M$ being of dimension $n+1$. We also denote by $\omega$ the scalar representative of $\Omega$ in a coordinate patch $\left(\mathcal{U} ; x^{i}, y, p_{i}\right)$.

Given an $n$-dimensional sub-manifold $N$ of $M$, we denote by $N_{1}$ the set of all the tangent spaces $T_{x} N$ with $x \in N$ (it being desirable to distinguish $N_{1}$ from TN). The set $N_{1}$ is an $n$-dimensional sub-manifold of $G(M)$ diffeomorphic to $N$ via the projection $\pi$ and is transversal to the fibres of $\pi$ i.e., $T_{H} N_{1} \cap T_{H} \pi^{-1}(x)=0$ for any $H=T_{x} N$. Manifolds such as $N_{1}$ will 
be called holonomic sub-manifolds of $G(M)$. The following lemma is easily verified, in local coordinates, using the form $\omega$.

Lemma 6.1. (i) The subspace $\Sigma_{H}=k e r_{H} \Omega$ is generated by all the contact elements $T_{H} N_{1}$ where $N_{1}$ is an arbitrary holonomic sub-manifold containing $H$. Equivalently, the canonical contact system $\mathcal{P}$ is generated by all the local 1-forms that vanish simultaneously on every holonomic submanifold.

(ii) The sub-space $\Sigma_{H}$ contains $T_{H} \pi^{-1}(x)$ where $x=\pi(H)$.

(iii) An n-dimensional sub-manifold $\mathcal{N}$ of $G(M)$ transversal to the fibres of $\pi$ is locally holonomic if and only if it is an integral manifold of $\mathcal{P}$ i.e., $\iota^{*} \Omega=0$ (or, equivalently, $\iota^{*} \omega=0$ ) where $\iota: \mathcal{N} \rightarrow G(M)$ is the inclusion map.

The property (i) shows that $\mathcal{P}$ admits, at any point, $n$-dimensional integral manifolds that are transversal to the fibres of $\pi$ and the property (ii) shows that each fibre $\pi^{-1}(x)$ is also an $n$-dimensional integral sub-manifold. There are however $n$-dimensional integral manifolds other than those mentioned above, for example the sub-manifold defined by the equations:

$$
x^{1}=\cdots=x^{n-1}=0, \quad y=x^{n}, \quad p_{n}=1 .
$$

More generally, let $N$ be a $q$-dimensional sub-manifold of $M, 0 \leq q \leq n$, and denote by $N_{1}$ the set of all the hyperplanes $H \in G(M)$ that are tangent to $\mathrm{N}$ namely, those verifying $T_{x} N \subset H$ where $x=\pi(H) \in N$. Clearly, $N_{1}$ is an $n$-dimensional sub-manifold of $G(M)$, the restricted projection $\pi: N_{1} \rightarrow N$ is a submersion and, since $\iota^{*} \Omega=0, \iota: N_{1} \rightarrow G(M)$, we infer that $N_{1}$ is also an integral sub-manifold of $\mathcal{P}$.

In particular, when $q=n, N_{1}$ becomes a holonomic sub-manifold and, when $q=0$, it is a discrete collection of fibres $\pi^{-1}(x), x \in N$. We now show that such sub-manifolds are, generically, all the $n$-dimensional integral manifolds of $\mathcal{P}$.

Lemma 6.2. Let $\mathcal{N}$ be an $n$-dimensional integral sub-manifold of $\mathcal{P}$ and let us assume that $\pi \mid \mathcal{N}$ has constant rank. Then $\mathcal{N}$ is, locally, a sub-manifold of the form $N_{1}$ with $\operatorname{dim} N=\operatorname{rank} \pi \mid \mathcal{N}$.

Proof. We can assume, without loss of generality, that $\pi(\mathcal{N})=N$ is a connected $q$-dimensional sub-manifold of $M$ and that $\pi: \mathcal{N} \rightarrow N$ is a submersion. Further, we can also assume that $0<q<n$ since the remaining two cases correspond precisely to the isolated fibres $\pi^{-1}(x)$ and to the holonomic sub-manifolds. Taking local coordinates $\left(\mathcal{U} ; x^{i}, y, p_{i}\right)$ on $G(M)$, the condition that $\omega=d y-p_{i} d x^{i}$ vanishes on $\mathcal{N}$ implies that $\iota^{*} d y$ is a linear 
combination of the restricted differentials $\iota^{*} d x^{i}$ where $\iota: \mathcal{N} \rightarrow G(M)$ is the inclusion map.

Moreover, since the projection $N$ is $q$-dimensional, we can choose, locally, $n-q$ functions among the $x^{i}$, say for convenience $\left\{x^{1}, \cdots, x^{n-q}\right\}$, such that the differentials $\iota^{*} d x^{i}=d\left(x^{i} \mid \mathcal{N}\right), 1 \leq i \leq n-q$, are linearly independent, the remaining differentials $\iota^{*} d x^{j}, j>n-q$, and $\iota^{*} d y$ being linear combinations of these. It then follows, always locally, that on $\mathcal{N}$, $x^{j} \mid \mathcal{N}=f^{j}\left(x^{1}\left|\mathcal{N}, \cdots, x^{n-q}\right| \mathcal{N}\right)$ and $y \mid \mathcal{N}=g\left(x^{1}\left|\mathcal{N}, \cdots, x^{n-q}\right| \mathcal{N}\right)$ hence, on $M$, the equations

$$
x^{j}-f^{j}\left(x^{i}\right)=0 \quad \text { and } \quad y-g\left(x^{i}\right)=0
$$

define a $q$-dimensional sub-manifold $N$. Finally, the vanishing of $\omega$ on $\mathcal{N}$ coupled with the local expressions of the functions $x^{j} \mid \mathcal{N}$ and $y \mid \mathcal{N}$, in terms of the $x^{i} \mid \mathcal{N}$, provide further $n-q$ independent equations

$$
\partial g / \partial x^{i}-\left(p_{i}+\sum p_{j} \partial f^{j} / \partial x^{i}\right)=0, \quad j>n-q,
$$

that, together with the equations (6.1), define locally the sub-manifold $\mathcal{N}$ and, for each $x \in N$, the equations (6.2) define the fibre $(\pi \mid \mathcal{N})^{-1}(x)$. It is now straightforward to verify that the equations (6.2) define precisely, in coordinates, the set of all the hyperplanes contained in $\mathcal{U}$ that are tangent to $N$. The proof is complete.

We also emphasize that the integral manifolds of $\mathcal{P}$ have at most the dimension $n$. In fact, we shall prove in the sequel that $(n+1)$-dimensional integral contact elements do not exist, such elements being those linear sub-spaces $W$ for which $\omega|W=d \omega| W=0$.

(a) If $W$ is transverse to the fibres of $\pi$, then the linear forms

$$
\left\{d x^{i}|W, d y| W\right\}, \quad 1 \leq i \leq n,
$$

are linearly independent hence $\omega \mid W \neq 0$.

(b) If $W \supset T_{H} \pi^{-1}(x)$, where $H$ is the base point of $W$, then

$$
v_{j}=\left(\partial / \partial p_{j}\right)_{H} \in W
$$

and consequently $i\left(v^{j}\right) d \omega=-d x^{j}$ vanishes on $W$. Since $\omega \mid W=0$, it also follows that $d y$ vanishes on $W$ hence this sub-space has at most dimension $n$. More generally, let $p=\operatorname{dim}\left(T_{H} \pi^{-1}(x) \cap W\right), p>0$, and let us denote by $\left(v^{1}, \cdots, v^{p}\right)$ a basis of the intersection.

Setting $v^{j}=\sum a_{i}^{j} \partial / \partial p_{i}$, the matrix $a_{i}^{j}$ has rank $p$ and therefore the linear forms $\mu^{j}=i\left(v^{j}\right) d \omega=-\sum a_{i}^{j} d x^{i}, 1 \leq j \leq p$, are independent, vanishing on $W$. We next consider the sub-space $V=T \pi(W) \subset T_{x} M$. Since $\operatorname{dim} V=n+1-p$ and since $d y\left|V=\sum p_{i} d x^{i}\right| V, p_{i}=p_{i}(H)$, it follows that a basis of $V^{*}$ can be chosen among the forms $\alpha^{i}=d x^{i} \mid V$, say 
$\left\{\alpha^{1}, \cdots, \alpha^{n+1-p}\right\}$. Taking vectors $w_{1}, \cdots, w_{n+1-p} \in W$ that satisfy the relations $\left\langle w_{k}, d x^{i}\right\rangle=\delta_{k}^{i}, 1 \leq i, k \leq n+1-p$, we can write

$$
w_{k}=\partial / \partial x^{k}+* \partial / \partial y+\sum * \partial / \partial p_{i}
$$

and, consequently,

$$
\eta_{k}=i\left(w_{k}\right) d \omega=i\left(w_{k}\right) \sum d x^{\ell} \wedge d p_{\ell} \equiv d p_{k} \bmod \left(d x^{1}, \cdots, d x^{n}\right) .
$$

We thus obtain a family of $n+1$ linearly independent 1 -forms $\left\{\mu^{j}, \eta_{k}\right\}$ vanishing on $W$, whereafter $\operatorname{dim} W \leq n$.

Remark 6.3. The above indicated proof is, in fact, an argument involving only the $2 n$-dimensional vector space $\Sigma_{H}$ and the exterior 2 -form $d \omega \mid \Sigma_{H}$ of maximum rank equal to $2 n$. Abstracting the specific context involving the Grassmannian space $G(M)$ as well as the Pfaffian system $\mathcal{P}$, it actually proves a well known result concerning Lagrangean linear sub-spaces.

Let $V$ be a $2 n$-dimensional real or complex vector space and $\bar{\omega}$ an exterior 2 -form of rank $2 n$ defined on $V$. Then the largest dimension of a linear subspace $W \subset V$ on which $\bar{\omega}$ vanishes is equal to $n$. This algebraic result, alone, limits the dimension of the integral sub-manifolds of $\mathcal{P}$.

We now turn our attention to the Cauchy problem for partial differential equations and, initially, restrict our discussion to first order systems since these already exhibit all the nuances of the theory and, to a great extent, simplify the language. By definition, a first order partial differential equations in one unknown function, on the manifold $M$, is a sub-manifold $\mathcal{S}$ of co-dimension 1 contained in $G(M)$.

To simplify the terminology we shall replace the full expression simply by differential equation and observe that, in the present context, it is far more befitting to consider contact elements rather than first order jets of local sections as is usually done. A solution of $\mathcal{S}$ is an $n$-dimensional submanifold $N$ of $M$ such that $N_{1} \subset \mathcal{S}$.

Taking coordinates $\left(\mathcal{U} ; x^{i}, y, p_{i}\right)$, the sub-manifold $\mathcal{S}$ can be described, locally, by means of an equation $F\left(x^{i}, y, p_{i}\right)=0$ and, if $N$ is the graph of a certain function $y=f\left(x^{1}, \cdots, x^{n}\right)$, this sub-manifold will be a solution of $\mathcal{S}$ if and only if the function $f$ is a solution of the partial differential equation

$$
F\left(x^{i}, y, \partial y / \partial x^{i}\right)=0 .
$$

We indicate by $\Omega \mid \mathcal{S}$ the restriction of $\Omega$ to the sub-manifold $\mathcal{S}$ i.e., the form $\iota^{*} \Omega$, where $\iota: \mathcal{S} \hookrightarrow G(M)$ is the inclusion, and by $\mathcal{P} \mid \mathcal{S}$ the Pfaffian system on the manifold $\mathcal{S}$ generated by $\Omega \mid \mathcal{S}$. It is, according to the general theory of partial differential equations, the canonical contact Pfaffian system restricted to the equation. 
We finally denote by $\Sigma \mid \mathcal{S}$ the annihilator of the aforementioned Pfaffian system, this annihilator being equal to the kernel of $\Omega \mid \mathcal{S}$ or, equivalently, the intersection $\Sigma \cap T \mathcal{S}$. Inasmuch, if $\omega$ denotes a local generator of $\mathcal{P}$, then its restriction $\iota^{*} \omega$ to $\mathcal{S}$ becomes a local generator of $\mathcal{P} \mid \mathcal{S}$.

To integrate the differential equation $\mathcal{S}$, in the sense given to this problem by Sophus Lie, consists in determining the $n$-dimensional integral submanifolds of the Pfaffian system $\mathcal{P} \mid \mathcal{S}$ and, of course, the integral manifolds corresponding to the solutions of the differential equation $\mathcal{S}$ will be precisely those sub-manifolds transverse to the fibres of $\pi$. In Section 7 we shall examine the integration procedure for such Pfaffian systems, as conceived by Sophus Lie, via the geometry of contact transformations. At present, we simply compute the class of $\mathcal{P} \mid \mathcal{S}$, the method being essentially the same as that employed for $\mathcal{P}$. In the paragraphs that follow, the symbol $*$ indicates a coefficient that needs not be written explicitly.

We observe initially that $\Omega \mid \mathcal{S}$ cannot vanish on any open subset $\mathcal{U}$ of $\mathcal{S}$ for then $\mathcal{U}$ would be a $2 n$-dimensional integral manifold of $\mathcal{P}$ which is never the case. However, since $\Omega \mid \mathcal{S}$ can eventually vanish on nowhere dense closed subsets, we shall assume that $\mathcal{P} \mid \mathcal{S}$ is a regular Pfaffian system on the whole manifold $\mathcal{S}$ in the sense that $(\Omega \mid \mathcal{S})_{H} \neq 0$ for all $H \in \mathcal{S}$ (i.e., the form $\Omega \mid \mathcal{S}$ never vanishes) or, equivalently, that

$$
T_{H} \mathcal{S}+\operatorname{ker} \Omega_{H}=T_{H} G(M) .
$$

We consider next the following two cases:

(a) $T_{H} \pi^{-1}(x) \subset T_{H} \mathcal{S}$. In this case, $v^{j}=\left(\partial / \partial p_{j}\right)_{H} \in(\Sigma \mid \mathcal{S})_{H}, 1 \leq j \leq n$, and $\operatorname{dim} T \pi\left(T_{H} \mathcal{S}\right)=n$ hence the rank of the linear forms

$$
i\left(v^{j}\right) d \omega\left|\mathcal{S}=-d x^{j}\right| \mathcal{S} \quad \text { and } \quad \omega|\mathcal{S} \equiv d y| \mathcal{S} \bmod \left(d x^{i} \mid \mathcal{S}\right)
$$

is precisely equal to $n$. Further, since

$$
T_{H} \pi^{-1}(x) \subset(\Sigma \mid \mathcal{S})_{H},
$$

then

$$
\operatorname{dim} T \pi\left[(\Sigma \mid \mathcal{S})_{H}\right]=n-1
$$

and we can choose vectors $w_{k} \in(\Sigma \mid \mathcal{S})_{H}, 1 \leq k \leq n-1$, as well as a sequence of indices $1 \leq i_{1}<\cdots<i_{n-1} \leq n$ in such a way that $\left\langle w_{k}, d x^{i_{\ell}}\right\rangle=\delta_{k}^{\ell}$. It follows that

$$
i\left(w_{k}\right) d \omega\left|\mathcal{S} \equiv\left(d p_{i_{k}}+* d p_{i_{n}}\right)\right| \mathcal{S} \bmod \left(d x^{i} \mid \mathcal{S}\right),
$$

where $i_{n}$ is the remaining index, whereafter the set of $2 n-1$ linearly independent 1-forms $\left\{i\left(v^{j}\right) d \omega\left|\mathcal{S}, i\left(w_{k}\right) d \omega\right| \mathcal{S}, \omega \mid \mathcal{S}\right\}$ generates the characteristic system $\tilde{\Delta}_{H}^{\perp}$ of $\mathcal{P} \mid \mathcal{S}$.

(b) $T_{H} \pi^{-1}(x) \not \subset T_{H} \mathcal{S}$. In this case,

$$
\operatorname{dim}\left(T_{H} \pi^{-1}(x) \cap T_{H} \mathcal{S}\right)=n-1,
$$


$T \pi\left(T_{H} \mathcal{S}\right)=T_{x} M$ and $\operatorname{dim} T \pi\left((\Sigma \mid \mathcal{S})_{H}\right)=n$.

Choosing a basis $\left\{v^{1}, \cdots, v^{n-1}\right\}$ of the intersection $T_{H} \pi^{-1}(x) \cap(\Sigma \mid \mathcal{S})_{H}$, we obtain $n-1$ linearly independent 1-forms $\mu^{j}=i\left(v^{j}\right) d \omega \mid \mathcal{S}$ since the $d x^{i} \mid \mathcal{S}$ are linearly independent on $\mathcal{S}$. Moreover, we can choose $n$ vectors $w_{k} \in(\Sigma \mid \mathcal{S})_{H}$ such that $\left\langle w_{k}, d x^{i}\right\rangle=\delta_{k}^{i}$ and therefore obtain further $n$ linear forms $\eta_{k}=i\left(w_{k}\right) d \omega \mid \mathcal{S}$. The dimension of the intersection being equal to $n-1$, the rank of the forms $d p_{k} \mid \mathcal{S}$ becomes also equal to $n-1$ hence the rank of the forms

$$
\begin{aligned}
\omega \mid \mathcal{S} & \equiv d y \mid \mathcal{S} \bmod \left(d x^{i} \mid \mathcal{S}\right), \\
\mu^{j} & \equiv 0 \bmod \left(d x^{i} \mid \mathcal{S}\right), \\
\eta_{k} & \equiv d p_{k} \mid \mathcal{S} \bmod \left(d x^{i} \mid \mathcal{S}\right)
\end{aligned}
$$

is at least equal to $2 n-1$. Since, by Lemma 5.1 , the rank of the characteristic system is always odd and since $\operatorname{dim} \mathcal{S}=2 n$, we infer that the above rank is constant and equal to $2 n-1$.

One can prove, by a straightforward though rather long calculation on the manifold $\mathcal{S}$, that the rank of the forms $\left\{\mu^{j}, \eta_{k}\right\}$ is, modulo $\omega \mid \mathcal{S}$, precisely equal to $2(n-1)$ thus obtaining the desired rank for the characteristic system. However, if we follow Cartan and compute on the space of all the variables, namely on $G(M)$, then it is quite easy to arrive at the desired conclusion and obtain, along the way, a very convenient representation of the characteristic system. Taking local coordinates $\left(\mathcal{U} ; x^{i}, y, p_{i}\right)$ on $G(M)$ and representing $\mathcal{S}$, locally, by the equation $F=0$, we observe that a vector $w \in T_{H} G(M)$, written as

$$
w=\sum u^{i} \partial / \partial x^{i}+a \partial / \partial y+\sum v_{i} \partial / \partial p_{i},
$$

belongs to $(\Sigma \mid \mathcal{S})_{H}$ if and only if

(i) $\langle w, \omega\rangle=0$, hence $a=\sum p_{i} u^{i}$ and

(ii) $\langle w, d F-\omega\rangle=0$, hence

$$
\sum\left(\frac{\partial F}{\partial x^{i}}+p_{i} \frac{\partial F}{\partial y}\right) u^{i}+\sum \frac{\partial F}{\partial p_{i}} v_{i}=0 .
$$

Since, by the regularity assumption on $\mathcal{P} \mid \mathcal{S}$, the forms $\omega$ and $d F$ are independent, the linear relation above is non-trivial and, furthermore, it is the only relation imposed on the coefficients $\left\{u^{i}, v_{i}\right\}$. We infer that the space of linear forms

$$
\left\{i(w) d \omega=\sum\left(u^{i} d p_{i}-v_{i} d x^{i}\right): w \in(\Sigma \mid \mathcal{S})_{H}\right\}
$$

has rank $2 n-1$ hence the linear system

$$
\left\{\omega, i(w) d \omega: w \in(\Sigma \mid \mathcal{S})_{H}\right\}
$$


has rank $2 n$, when considered on the manifold $G(M)$, and consequently its kernel $V_{H}$ has dimension equal to 1 .

Moreover, since the linear sub-space $(\Sigma \mid \mathcal{S})_{H}$ is odd-dimensional, the restricted 2-form $d \omega \mid(\Sigma \mid \mathcal{S})_{H}$ has a non-trivial annihilator that is clearly equal to $V_{H}$ hence the characteristic space $(\tilde{\Delta})_{H}$ of $\mathcal{P} \mid \mathcal{S}$ is also equal to $V_{H}$ and the restricted system

$$
\left\{\omega|\mathcal{S}, i(w) d \omega| \mathcal{S}: w \in(\Sigma \mid \mathcal{S})_{H}\right\},
$$

on the manifold $\mathcal{S}$, has rank $2 n-1$ as desired. The characteristic distribution $\tilde{\Delta}=\Delta$ being of dimension 1 , it reduces to a system of ordinary differential equations. The relations

$$
\sum u^{i} d p_{i}-v_{i} d x^{i}=0 \quad \text { and } \quad d y-\sum p_{i} d x^{i}=0
$$

together with (6.4) provide the system:

$$
\begin{aligned}
\frac{d x^{1}}{\partial F / \partial p_{1}}=\frac{d x^{2}}{\partial F / \partial p_{2}}= & \cdots \frac{d x^{n}}{\partial F / \partial p_{n}}=\frac{-d p_{1}}{\partial F / \partial x^{1}+p_{1} \partial F / \partial y}= \\
& =\cdots=\frac{-d p_{n}}{\partial F / \partial x^{n}+p_{n} \partial F / \partial y}=\frac{d y}{\Sigma p_{i} \partial F / \partial p_{i}}
\end{aligned}
$$

on the manifold $G(M)$, corresponding to the distribution $H \mapsto V_{H}$, hence its restriction to $\mathcal{S}$ provides the Cartan characteristics of the Pfaffian system $\mathcal{P} \mid \mathcal{S}$. These characteristics coincide, in view of the equation (6.5), with the well known characteristic stripes of the partial differential equation (6.3) i.e., with the Cauchy characteristics of this equation. The system (6.5) applies as well to the case (a) since neither assumptions (a) nor (b) are relevant in Cartan's argument. It should however be observed that the case (b) is, in fact, the natural context for the partial differential equation (6.3).

The previous results extend easily to systems of partial differential equations. By definition, such a system of order 1 on the manifold $M$ (and in one unknown function) is a sub-manifold $\mathcal{S}$ of $G(M)$. When codim $\mathcal{S}=q$, this sub-manifold is locally defined by a system of $q$ independent equations $\left\{F_{\alpha}=0\right\}$, these being called the local equations of $\mathcal{S}$. A solution of $\mathcal{S}$ is an $n$-dimensional sub-manifold $N$ of $M$ such that $N_{1} \subset \mathcal{S}$ hence, if we are concerned in studying integrability problems, the systems of co-dimension $q>n+1$ become irrelevant.

We shall therefore assume that $q \leq n+1$, the integer $q$ being called the rank of the system $\mathcal{S}$. Taking local coordinates $\left(\mathcal{U} ; x^{i}, y, p_{i}\right)$, the single relation (6.3) is now replaced by

$$
F_{\alpha}\left(x^{i}, y, \partial y / \partial x^{i}\right)=0, \quad 1 \leq \alpha \leq q .
$$

Similarly, we introduce the restricted form $\Omega \mid \mathcal{S}$, the restricted Pfaffian system $\mathcal{P} \mid \mathcal{S}$, the restricted distribution $\Sigma \mid \mathcal{S}$ and the restricted generator $\omega \mid \mathcal{S}$. 
We also restrict our attention to those equations $\mathcal{S}$ providing regular Pfaffian systems $\mathcal{P} \mid \mathcal{S}$ in the sense that they are locally trivial sub-bundles of $T^{*} \mathcal{S}$ and call such equations regular. When $q \leq n$, the restricted form $\Omega \mid \mathcal{S}$ cannot vanish on any open subset $\mathcal{U}$ of $\mathcal{S}$ otherwise this open set would be an integral manifold of $\mathcal{P}$ of dimension greater than $n$. We infer that the regularity of $\mathcal{P} \mid \mathcal{S}$, when $q \leq n$, simply means that $(\Omega \mid \mathcal{S})_{H} \neq 0$ for any $H \in \mathcal{S}$. However, when $q=n+1, \mathcal{P} \mid \mathcal{S}$ can be the null system as is necessarily the case when it admits solutions.

We say that $\mathcal{S}$ is integrable when every point $H \in \mathcal{S}$ belongs to an $n$ dimensional integral manifold of the Pfaffian system $\mathcal{P} \mid \mathcal{S}$ and now prove that such a system has regular characteristics. If $q=n+1$, integrability of $\mathcal{S}$ implies that $\mathcal{P} \mid \mathcal{S}=0$, the null system admitting regular characteristics since the manifold $\mathcal{S}$ is a characteristic. Moreover, $\mathcal{S}$ is locally a holonomic sub-manifold of $G(M)$.

Theorem 6.4. Let $\mathcal{S}$ be a system of regular and integrable first order partial differential equations of rank $q<n+1$ defined on the manifold $M$. Then the associated Pfaffian system $\mathcal{P} \mid \mathcal{S}$, on the manifold $\mathcal{S}$, has regular characteristics and its class is equal to $2(n-q)+1$.

Proof. We follow Cartan's argument and begin by taking a local coordinate system $\left(\mathcal{U} ; x^{i}, y, p_{i}\right)$ on $G(M)$ as well as local equations $F_{\alpha}=0$ for $\mathcal{S}$. Since the forms $\left\{\omega, d F_{\alpha}\right\}$ are linearly independent, the equation (6.4) is replaced by a system of independent equations

$$
\sum\left(\frac{\partial F_{\alpha}}{\partial x^{i}}+p_{i} \frac{\partial F_{\alpha}}{\partial y}\right) u^{i}+\sum \frac{\partial F_{\alpha}}{\partial p_{i}} v_{i}=0
$$

hence the kernel $V_{H}$ of the system $\left\{\omega, i(w) d \omega: w \in(\Sigma \mid \mathcal{S})_{H}\right\}$ has dimension q. Further, the characteristic sub-space $\tilde{\Delta}_{H}$ of $\mathcal{P} \mid \mathcal{S}$ being necessarily contained in $V_{H}$, it suffices to show, as a consequence of the integrability, that $\operatorname{dim} \tilde{\Delta}_{H} \geq q$. In fact, $\mathcal{S}$ being integrable, the linear space $(\Sigma \mid \mathcal{S})_{H}$ contains an $n$-dimensional sub-space $W$ on which $d \omega$ vanishes.

Let us write $\mu=d \omega \mid(\Sigma \mid \mathcal{S})_{H}$. Then, since $\operatorname{dim}(\Sigma \mid \mathcal{S})_{H}=2 n-q$, we can choose a basis $\left\{v_{1}, \cdots, v_{2 n-q}\right\}$ of $(\Sigma \mid \mathcal{S})_{H}$ in such a way that $v_{j} \in W$ for $j \leq n$ and consequently the characteristic sub-space $\tilde{\Delta}_{H}$ becomes the kernel of the linear forms $\left\{i\left(v_{k}\right) \mu: 1 \leq k \leq 2 n-q\right\}$. Observing now that $\mu \mid W=0$, we infer that $i\left(v_{j}\right) \mu \mid W=0$ for $j \leq n$ and that the forms $\left\{i\left(v_{k}\right) \mu \mid W: k>n\right\}$ vanish on a sub-space of dimension at last equal to $n-(2 n-q-n)=q$ hence $\operatorname{dim} \tilde{\Delta}_{H} \geq q$ as desired. The class of $\mathcal{P}$ is therefore equal to $(2 n+1-q)-q=2(n-q)+1$. 
We terminate this section by discussing the integration procedure of the system $\mathcal{P} \mid \mathcal{S}$ via its characteristics. We integrate, in the first place, the characteristic distribution so as to obtain, locally, the characteristic variables of $\mathcal{P} \mid \mathcal{S}$.

In the Part II, we shall discuss an algorithm especially devised for this purpose. Next and according to the Corollary 4.2, we reduce locally the system $\mathcal{P} \mid \mathcal{S}$ to the space of is characteristic variables and observe, in view of Lemma 5.4, that the quotient system $\tilde{\mathcal{P}} \mid \mathcal{S}$ is an odd classical contact structure defined on a space of dimension $2(n-q)+1$.

It will then suffice to take an $(n-q)$-dimensional integral manifold $\tilde{\mathcal{V}}$ of $\tilde{\mathcal{P}} \mid \mathcal{S}$, this being achieved by reducing $\tilde{\mathcal{P}} \mid \mathcal{S}$ to its canonical form $\tilde{\mu}=0$ with $\tilde{\mu}=d Z-\sum P_{i} d X^{i}, 1 \leq i \leq n-q$, (recall the previous discussion concerning the integral manifolds of the canonical contact structure $\mathcal{P}$ on $G(M))$ and lift it to an integral manifold $\mathcal{V}=\rho^{-1}(\tilde{\mathcal{V}})$ of $\mathcal{P} \mid \mathcal{S}$, the dimension of $\mathcal{V}$ being equal to $(n-q)+q=n$. From a practical point of view, the above discussion boils down to finding a generator $\mu$ of class $2(n-q)+1$ for the system $\mathcal{P} \mid \mathcal{S}$, expressed in canonical form.

The above integration procedure also provides, as an extra dividend, a result that puts in evidence the full significance of the characteristics.

Corollary 6.5. Let $\mathcal{S}$ be a regular system of partial differential equations of rank $q \leq n+1$ on the manifold $M$ and let us assume that the associated contact Pfaffian system $\mathcal{P} \mid \mathcal{S}$ has regular characteristics of dimension equal to inf $\{q, n\}$. Then $\mathcal{S}$ is integrable.

Regularity of the characteristics together with the appropriate dimension is therefore a necessary and sufficient condition for integrability.

\section{Contact transformations}

In this section we study more carefully the geometry of the contact structure defined on the Grassmannian bundle $G(M)$ by the fundamental form $\Omega$. Several results are generic statements concerning odd dimensional classical contact structures and could as well be developped in the general context, others are more specific to the structure on $G(M)$.

Let $\mathcal{P}$ be the canonical contact structure on $G(M)$. The finite automorphisms of $\mathcal{P}$ are called the (first order) contact transformations and the infinitesimal automorphisms the infinitesimal contact transformations or Lie vector fields. In local coordinates $\left(\mathcal{U} ; x^{i}, y, p_{i}\right)$, contact transformations $\varphi$ are defined by the equation $\varphi * \omega \equiv \omega \bmod \omega$ and Lie vector fields $\xi$ by $\theta(\xi) \omega \equiv \omega \bmod \omega$.

Among all the contact transformations, the simplest are those that arise from the transformations on the base space $M$. Any local diffeomorphism 
$\varphi: U \rightarrow U^{\prime}$ of $M$ operates, via $T \varphi$, on the linear contact elements of $M$ defining thereafter a local diffeomorphism $\wp \varphi: \pi^{-1}(U) \rightarrow \pi^{-1}\left(U^{\prime}\right)$ on $G(M)$ by setting $\wp \varphi\left(H_{x}\right)=T \varphi\left(H_{x}\right)$. The first statement in Lemma 6.1 assures that $\wp \varphi$ is a contact transformation. The assignment $\varphi \mapsto \wp \varphi$ is obviously functorial i.e.,

$$
\wp \mathrm{Id}=\mathrm{Id}, \quad \wp(\psi \circ \varphi)=\wp \psi \circ \wp \varphi, \quad \wp\left(\varphi^{-1}\right)=(\wp \varphi)^{-1}
$$

and local i.e., $\wp\left(\cup \varphi_{\alpha}\right)=\cup \wp \varphi_{\alpha}$ where $\cup \varphi_{\alpha}$ denotes the transformation obtained by patching together the transformations $\varphi_{\alpha}$ that agree on the overlaps.

We infer, in particular, that a differentiable 1-parameter family $\left(\varphi_{t}\right)$ of local diffeomophisms of $M$ (resp. a local 1-parameter group) gives rise to a differentiable 1-parameter family $\left(\wp \varphi_{t}\right)$ on $G(M)$ (resp. a local 1- parameter group) hence any local vector field $\xi$, defined on an open set $U$ of $M$ and generating the local 1-parameter group $\left(\varphi_{t}\right)$, gives rise to the Lie vector field $\wp \xi=\left.\frac{d}{d t} \wp \varphi_{t}\right|_{t=0}$ defined on the open set $\pi^{-1}(U)$. The assignment $\xi \mapsto \wp \xi$ is, of course, local with respect to the patching of vector fields. Moreover, since $\wp \xi$ can as well be defined by the above derivative where $\left(\varphi_{t}\right)$ is replaced by any differentiable 1-parameter family satisfying $\varphi_{0}=\operatorname{Id}$ and $\left.\frac{d}{d t} \varphi_{t}\right|_{t=0}=\xi$, and since

$$
\begin{gathered}
\xi+\eta=\left.\frac{d}{d t} \varphi_{t} \circ \psi_{t}\right|_{t=0}, \\
\alpha \xi=\left.\frac{d}{d t} \varphi_{\alpha t}\right|_{t=0}, \quad \alpha \in \mathbf{R}, \\
{[\xi, \eta]=\left.\frac{d}{d t} \varphi_{-s} \psi_{-s} \varphi_{s} \psi_{s}\right|_{t=0}, \quad s=|t|^{1 / 2},}
\end{gathered}
$$

we infer that the above assignment is functorial with respect to the Lie algebra sheaf structure i.e.,

$$
\wp(\xi+\eta)=\wp \xi+\wp \eta, \quad \wp(\alpha \xi)=\alpha \wp \xi, \quad \wp[\xi, \eta]=[\wp \xi, \wp \eta] .
$$

It should be observed, however, that $\wp(f \xi)$ is not equal to $f \wp \xi$ unless $f$ is a constant function and the general expression is, of course,

$$
\wp(f \xi)=f \wp \xi+\delta(f) \xi
$$

where $\delta$ is a certain Lie derivative. The transformations $\wp \varphi$ and $\wp \xi$ are called the prolongations of $\varphi$ and $\xi$, respectively, to the bundle $G(M)$ and project, via $\pi$, onto the initial transformations $\left(\pi \circ \wp \varphi=\varphi \circ \pi\right.$ and $\left.\pi_{*} \wp \xi=\xi\right)$.

Lemma 7.1. A necessary and sufficient condition that a contact transformation $\varphi$ (resp. a Lie vector field $\xi$ ) be the prolongation of a base transformation (resp. a base vector field) is that it projects, via $\pi$, onto a local diffeomorphism $\varphi_{0}$ (resp. onto a local vector field $\xi_{0}$ ) of $M$. This being the case, then $\varphi=\wp \varphi_{0}$ (resp. $\xi=\wp \xi_{0}$ ) on the common domain. 
The proof will be omitted since it is obvious.

We consider again, as in Section 5 , the line bundle $\Phi$ on the manifold $G(M)$ and define, for each Lie vector field $\xi$, Section $h_{\xi}=\langle\xi, \Omega\rangle$ of $\Phi$ whose domain coincides with that of $\xi$. The section $h_{\xi}$ is called the contact hamiltonian of $\xi$. According to the notations of Section 5, we denote by $\mathcal{L}(\mathcal{P})$ the set of all the Lie vector fields, $\mathcal{P}$ being the canonical contact structure on the manifold $G(M)$, and by $\Gamma_{\ell}(\Phi)$ the set of all the local sections of $\Phi$.

Lemma 7.2. The mapping $\mathcal{L}(\mathcal{P}) \rightarrow \Gamma_{\ell}(\Phi), \xi \mapsto h_{\xi}$, is bijective.

Proof. We first argue locally. Taking a coordinate system $\left(\mathcal{U} ; x^{i}, y, p_{i}\right)$ on $G(M)$, we consider the local section $[\partial / \partial y]=\partial / \partial y \bmod \mathcal{E}(c f$. the Example 5.7) of the bundle $\Phi$, induced by the vector field $\partial / \partial y$ on $G(M)$. This section is everywhere non-null and therefore trivializes the bundle $\Phi$ on the open set $\mathcal{U}$. Since $\Omega \mid \mathcal{U}=\omega[\partial / \partial y]$, the hamiltonian $h_{\xi}=\langle\xi, \omega\rangle[\partial / \partial y]$ can be replaced by the scalar function $H_{\xi}=\langle\xi, \omega\rangle$ called the hamiltonian of $\xi$ relative to $\omega$. Furthermore, since $\xi$ is an infinitesimal automorphism of $\mathcal{P}$, then $\theta(\xi) \omega=i(\xi) d \omega+d i(\xi) \omega=\lambda \omega$ hence $\left(i(\xi) d \omega+d H_{\xi}\right) \wedge \omega=0$. A straightforward computation will also show that, conversely, given any differentiable function $f$, the equations

$$
i(\xi) \omega=f \quad \text { and } \quad(d f+i(\xi) d \omega) \wedge \omega=0
$$

have a unique solution $\xi$, this solution being, of course, a Lie vector field since the second equation is simply $[\theta(\xi) \omega] \wedge \omega=0$. More precisely, the coordinate expression of $\xi$ is given by

$$
\xi=-\sum \frac{\partial f}{\partial p_{i}} \frac{\partial}{\partial x^{i}}+\left(f-\sum \frac{\partial f}{\partial p_{i}} p_{i}\right) \frac{\partial}{\partial y}+\sum\left(\frac{\partial f}{\partial x^{i}}+p_{i} \frac{\partial f}{\partial y}\right) \frac{\partial}{\partial p_{i}} .
$$

The injectivity of the mapping in Lemma 7.2 is now obvious since, assuming that $h_{\xi}=0$, the scalar hamiltonian $H_{\xi}$, defined on any coordinate patch, also must vanish and therefore $\xi=0$. We observe that the injectivity follows inasmuch from the fact that the system $\mathcal{P}$ has null characteristics. If $f=\langle\xi, \omega\rangle=0$, then $\xi$ is a characteristic vector field of $\mathcal{P}$ and consequently $\xi=0$. In order to prove the surjectivity, we take a local section $\sigma$ of $\Phi$ defined on an open set $U$, a coordinate system $\left(\mathcal{U} ; x^{i}, y, p_{i}\right)$, trivialize the bundle $\Phi \mid \mathcal{U}$ by means of the section $[\partial / \partial y]$ and consider the unique solution $\xi$ of the equations (7.1), where $f$ and $\omega$ are determined respectively by $\sigma \mid \mathcal{U}=f[\partial / \partial y]$ and $\Omega \mid \mathcal{U}=\omega[\partial / \partial y]$ restricted to the open set $\mathcal{U} \cap U$. We claim that two such solutions $\xi$ and $\bar{\xi}$, defined via two coordinate systems $(\mathcal{U} ; x, y, p)$ and $(\overline{\mathcal{U}} ; \bar{x}, \bar{y}, \bar{p})$, do agree on the overlap, defining thereafter a unique Lie vector field on the open set $U$ whose hamiltonian is the given 
section $\sigma$. We leave the details of the proof to the reader since it only involves straightforward calculations.

The Lie bracket defined in $\mathcal{L}(\mathcal{P})$ can now be transported, via the isomorphism of Lemma 7.2, to a Lie algebra bracket on the space $\Gamma_{\ell}(\Phi)$ namely, the Lagrange bracket for contact hamiltonians. Given a coordinate system $(\mathcal{U} ; x, y, p)$, hamiltonians can be replaced by differentiable functions and the Lagrange bracket becomes a Lie algebra bracket on functions. If $\xi$ is a Lie vector field and $f=\langle\xi, \omega\rangle$ the corresponding hamiltonian, then it is easy to verify that

$$
[f, g]=\xi g-g \partial f / \partial y
$$

We next observe that

$$
\langle\xi, d f\rangle=\langle\xi, d f+i(\xi) d \omega\rangle=\langle\xi, \lambda \omega\rangle=\lambda f,
$$

hence $\langle\xi, d f\rangle_{H}=0$ whenever $f(H)=0$ and of course, under these conditions, $\langle\xi, \omega\rangle_{H}=0$. Expressed in other terms, the Lie vector field $\xi$ with hamiltonian $h \in \Gamma_{\ell}(\Phi)$ is tangent to the variety $h^{-1}(0)$ and, at these points, takes values in the contact structure $\Sigma$. We observe that $h^{-1}(0)$ needs not be a regular sub-manifold of $G(M)$ since the function $f$ can admit singularities. Nevertheless, for any $H \in h^{-1}(0)$, the curve $\exp t \xi(H)$ is entirely contained in $h^{-1}(0)$ since the relation $\langle\xi, d f\rangle=\lambda f$ is equivalent to $\xi f=\lambda f$ and therefore equivalent, when $H \in G(M)$ is fixed, to the linear homogeneous equation

$$
\frac{d}{d t} f(\exp t \xi(H))=\lambda(\exp t \xi(H)) f(\exp t \xi(H)),
$$

in the unknown function $f(\exp t \xi(H))$. If $H \in h^{-1}(0)$, then

$$
f(H)=0=f(\exp 0 \xi(H)),
$$

hence $f(\exp t \xi(H)) \equiv 0$. We infer that $\exp t \xi(H)$ is an integral curve of the system $\mathcal{P}$ contained in the level variety $h^{-1}(0)$.

Lemma 7.3. Let $\xi$ be a Lie vector field with hamiltonian $h$ and $\mathcal{N}$ a regularly embedded sub-manifold of $G(M)$ contained in $h^{-1}(0)$. Let us further assume that $\mathcal{N}$ is an integral manifold of $\mathcal{P}$ (i.e., $\Omega \mid \mathcal{N}=0$ ) and that $\xi_{H} \notin T_{H} \mathcal{N}$ for any $H \in \mathcal{N}$ (i.e., the vector field $\xi$ is transversal to $\mathcal{N}$ ). Under these assumptions, the union of all the flow lines of $\xi$ containing points of $\mathcal{N}$ is, for small values of $t$, a regularly embedded integral submanifold of $\mathcal{P}$. More precisely, we can choose, for any $H \in \mathcal{N}$, an open neighborhood $\mathcal{V}_{H} \subset \mathcal{N}$ and a constant $\epsilon_{H}$ such that $\cup_{H} \cup_{t, K} \exp t \xi(K)$, $H \in \mathcal{N}, K \in \mathcal{V}_{H},|t|<\epsilon_{H}$, is an integral manifold $\widetilde{\mathcal{N}}$ of $\mathcal{P}$ whose dimension is equal to $\operatorname{dim} \mathcal{N}+1$. 
Proof. It will suffice to argue locally and glue together the local integral manifolds thus obtained. Let us take a point $H \in \mathcal{N}$ and choose appropriately a neighborhood $\mathcal{V}$ of $H$ in $\mathcal{N}$ as well as a constant $\epsilon>0$ such that $\exp t \xi(K), K \in \mathcal{V},|t|<\epsilon$, is defined. The transversality condition on $\xi$ implies that the mapping $] \epsilon, \epsilon[\times \mathcal{V} \rightarrow G(M),(t, K) \mapsto \exp t \xi(K)$, is an immersion for small $T$ whereupon its image is a regularly embedded submanifold for, eventually, a smaller constant $\epsilon$ and a smaller neighborhood $\mathcal{V}$. We can therefore assume that the image is in fact a sub-manifold $\tilde{\mathcal{N}}$ that is furthermore contained in $h^{-1}(0)$ since $H$ is the hamiltonian of $\xi$ and $\mathcal{V} \subset h^{-1}(0)$. To prove that $\widetilde{\mathcal{N}}$ is an integral sub-manifold of $\mathcal{P}$, we first observe that the curves $\exp t \xi(K)$ are integral curves of $\mathcal{P}$ and, since $\xi$ is an infinitesimal automorphism of this Pfaffian system, we infer that $\mathcal{V}_{t}=\exp t \xi(\mathcal{V})$ is, for any fixed value of $t$, an integral manifold of $\mathcal{P}$. We next observe that the tangent space to $\widetilde{\mathcal{N}}$, at any point $L=\exp t \xi(K)$, is generated by the tangent space $T_{L} \mathcal{V}_{t}$ together with the vector $\xi_{L}$ tangent to the curve $\exp t \xi(K)$. It then follows readily that the form $\Omega$ vanishes on $T_{L} \widetilde{\mathcal{N}}$ and therefore that $\widetilde{\mathcal{N}}$ is an integral manifold of $\mathcal{P}$.

We now examine more closely the flow of the Lie vector field $\xi$ with hamiltonian $h$ (or $f$ ). From the local expression (7.2), we infer that $\xi_{H}=0$ if and only if

$$
f(H)=\frac{\partial f}{\partial p_{i}}(H)=\left(\frac{\partial f}{\partial x^{i}}+p_{i} \frac{\partial f}{\partial y}\right)(H)=0,
$$

a result that can be obtained as well, from the equation (7.1), as follows: we observe that these equations provide pointwise conditions on the vector $\xi_{H}$ namely, given any function $f$ and any point $H \in G(M)$, there exists a unique vector $v \in T_{H} G(M)$ for which

$$
i(v) \omega_{H}=f(H) \quad \text { and } \quad d f_{H}+i(v) d \omega_{H}=0 .
$$

In fact, let $v^{\prime}$ be a second vector satisfying the above condition. Then $i\left(v-v^{\prime}\right) \omega_{H}=0$ i.e., $\left(v-v^{\prime}\right) \in \Sigma_{H}$ and $\left(i\left(v-v^{\prime}\right) d \omega_{H}\right) \wedge \omega_{H}=0$ hence $\left(v-v^{\prime}\right)=0$ since $d \omega_{H} \mid \Sigma_{H}$ has maximal rank. We infer that $\xi_{H}=0$ if and only if

$$
f(H)=0 \quad \text { and } \quad d f_{H} \wedge \omega_{H}=0 \quad\left(\text { or }\left(i\left(\xi_{H}\right) d \omega_{H}\right) \wedge \omega_{H}=0\right),
$$

the later condition asserting that $\operatorname{ker} d f_{H} \supset \operatorname{ker} \omega_{H}=\Sigma_{H}$. Therefore, if $d f_{H} \wedge \omega_{H} \neq 0$ everywhere, the flow lines of $\xi$ are 1-dimensional submanifolds of $G(M)$. Replacing now the function $f$ by $g f$, the equations $i(g \xi) \omega=(g f)(H)$ and $(d(g f)+i(g \xi) d \omega) \wedge \omega=0$ are satisfied at any point $H \in f^{-1}(0)$ hence the Lie vector field $\eta$ with hamiltonian $g f$ coincides with $g \xi$ along the variety $h^{-1}(0)$. When $g$ is everywhere non-zero, the flow 
lines of $\xi$ and $g \xi$ provide the same 1-dimensional sub-manifolds, the flows differing only by their parametrizations.

Coming back to partial differential equations of order 1 , we defined them in Section 6 as being sub-manifolds $\mathcal{S}$ of co-dimension 1 in $G(M)$ and assumed that $\mathcal{P} \mid \mathcal{S}$ is a regular Pfaffian system on this same sub-manifold. Equivalently, this amounts to say that the restricted fundamental form $\Omega \mid \mathcal{S}$ never vanishes. Such a sub-manifold is defined locally by a non-singular equation $f=0$ (i.e., $d f_{H} \neq 0$ for any $H \in \mathcal{S}$ ), any other non-singular equation, in the neighborhood of the same point $H_{0}$, being of the form $g f=0$ with $g(H) \neq 0$ for any $H \in \mathcal{S}$. Such a local equation $f=0$ gives rise to a Lie vector field $\xi$ on $G(M)$ with hamiltonian equal to $f$, this vector field being tangent to the sub-manifold $\mathcal{S}$ and consequently providing an infinitesimal automorphism of the Pfaffian system $\mathcal{P} \mid \mathcal{S}$.

Furthermore, since $\langle\xi, \omega\rangle=f$ vanishes on $\mathcal{S}$ (locally equal to $f^{-1}(0)$ ), we infer that $\xi \mid \mathcal{S}$ is a characteristic vector field of $\mathcal{P} \mid \mathcal{S}$. Finally, since the regularity condition on $\mathcal{P} \mid \mathcal{S}$ simply means that $(d f \wedge \omega)_{H}, H \in \mathcal{S}$, is everywhere non-zero and since the Cauchy characteristics of $\mathcal{P} \mid \mathcal{S}$ are 1-dimensional submanifolds, we infer that the vector field $\xi \mid \mathcal{S}$ is also everywhere non-zero and that its flow lines are open subsets of the characteristics, any other local non-singular equation $\tilde{f}=g f=0$ providing the same flow lines.

In conclusion, the Cauchy characteristics of $\mathcal{P} \mid \mathcal{S}$ are unions of flow lines of Lie vector fields whose hamiltonians arise from local regular equations for the sub-manifold $\mathcal{S}$.

We can now restate, in the context of contact transformations, the results of section 6 concerning the Cauchy characteristics of a single equation.

Theorem 7.4. Let $\mathcal{S}$ be a first order partial differential equation on the manifold $M$, denote by $\xi$ the Lie vector field with hamiltonian $f$ and assume that $f=0$ is a regular local equation of $\mathcal{S}$. Then $\xi$ is tangent to $\mathcal{S}$ and the flow lines of the restricted vector field $\xi \mid \mathcal{S}$ are open subsets of the (1-dimensional) Cauchy characteristics of $\mathcal{P} \mid \mathcal{S}$, independently of the particular choice of the hamiltonian $f$. Any maximal $(=n)$ dimensional integral manifold of the Pfaffian system above is the union of such flows and, given any $(n-1)$-dimensional regularly embedded integral sub-manifold $\mathcal{N}$ of $\mathcal{P} \mid \mathcal{S}$ that is transversal to the Cauchy characteristics, it can be extended to an n-dimensional integral manifold by taking the union of all the flow lines of $\xi$ beginning at the points of $\mathcal{N}$.

It is often useful to handle a version of the previous theorem when the function $f$ is replaced by the hamiltonian $h$. With this in mind, we claim that it suffices to determine conditions under which the equation $h=0$ is equivalent to a regular local equation $f=0$, where $h$ is a local section 
of the line bundle $\Phi, \xi$ the Lie vector field with hamiltonian $h=\langle\xi, \Omega\rangle$, $f=\langle\xi, \omega\rangle$ and $h=f[\partial / \partial y]$.

We recall ([6]) that, given any vector bundle $E$ with base manifold $M$ and projection $\pi$, the following short sequence is exact:

$$
0 \rightarrow T^{*} M \otimes E \stackrel{\iota}{\rightarrow} J_{1} E \stackrel{\beta}{\rightarrow} E \rightarrow 0,
$$

where $J_{1} E$ is the first order jet bundle of the local sections of $\pi: E \rightarrow M$. Each 1-jet identifies with an $n$-dimensional linear contact element of $\mathrm{E}$, transverse to the fibres of $\pi(n=\operatorname{dim} M)$. In particular, the line bundle $\Phi$ with base space $G(M)$ provides the following short exact sequence:

$$
0 \rightarrow T^{*} G(M) \otimes \Phi \stackrel{\iota}{\rightarrow} J_{1} \Phi \stackrel{\beta}{\rightarrow} \Phi \rightarrow 0 .
$$

Given a hamiltonian $h \in \Gamma_{\ell}(\Phi)$, its image $\mathcal{H}$ is a sub-manifold of $\Phi$ and therefore $\mathcal{H}_{1}=\cup T_{y} \mathcal{H}, y \in \mathcal{H}$, is also a sub-manifold of $J_{1} \Phi$. The set of points $H \in G(M)$ where $h$ vanishes is equal to the projection, in $G(M)$, of $\mathcal{H} \cap O_{\Phi}$, the second term being the null section, hence at these points, $\beta\left(T_{h(H)} \mathcal{H}\right)=0$ and therefore $T_{h(H)} \mathcal{H}=\iota\left(u_{H}\right)$ with $u_{H} \in T^{*} G(M) \otimes \Phi$. It is easy to check that $h=0$ is a regular equation if and only if $u_{H} \neq 0$ at any point $H \in G(M)$ where $h(H)=0$.

We now extend the previous results to systems of partial differential equations and state, firstly, some auxiliary lemmas, establishing thereafter the desired results. The remark following the relation (7.4) and the fact that a linear combination, with constant coefficients, of Lie vector fields is again a Lie vector field implies the following

Lemma 7.5. Let $\left\{\xi_{\alpha}\right\}$ be a family of Lie vector fields with associated hamiltonians $\left\{f_{\alpha}\right\}$, consider a fixed element $H \in G(M)$ and assume that the family $\left\{\left(d f_{\alpha} \wedge \omega\right)_{H}\right\}$ is linearly independent. Then the family $\left\{\xi_{\alpha}(H)\right\}$ is also linearly independent and, if moreover $f_{\alpha}(H)=0$ for all $\alpha$, the above sufficient condition becomes as well necessary.

Using the local expression of the Lagrange bracket and an argument involving linear homogeneous ordinary differential equations similar to that preceding Lemma 7.3, we also derive the following result.

Lemma 7.6. Let $\left\{\xi_{\alpha}\right\}$ be a family of Lie vector fields with associated hamiltonians $\left\{f_{\alpha}\right\}$, and let $\left\{g_{\beta}\right\}$ be an arbitrary family of local functions on $G(M)$. In order that the family of vector fields $\left\{\xi_{\alpha}\right\}$ be tangent to the variety $\mathcal{W}$ defined by the equations $g_{\beta}=0$ (i.e., $\left\langle\xi, d g_{\beta}\right\rangle=0$ ) it is necessary and sufficient that $\left[f_{\alpha}, g_{\beta}\right] \mid \mathcal{W}=0$ for all the indices $\alpha$ and $\beta$. Under these conditions and restricting our attention to finite subsets of indices $\alpha$, the 
image of the exponential map $\left(t^{\alpha}\right) \mapsto \exp \left(\sum t^{\alpha} \xi_{\alpha}\right)(H)$ initiating at points $H \in \mathcal{W}$ is entirely contained in $\mathcal{W}$.

Again, the remark following the relation (7.4) and the local expression of the Lagrange bracket yield the following two lemmas.

Lemma 7.7. Let $\left\{f_{\alpha}\right\}$ be a finite family of independent functions defined locally on $G(M),\left\{\xi_{\alpha}\right\}$ the corresponding family of Lie vector fields, $\mathcal{W}$ the regularly embedded sub-manifold defined by the equations $\left\{f_{\alpha}=0\right\},\left\{g_{\beta}\right\}$ an arbitrary family of functions vanishing on $\mathcal{W}$ and $\left\{\eta_{\beta}\right\}$ the corresponding family of Lie vector fields. Under these conditions:

(a) Each $\eta_{\beta} \mid \mathcal{W}$ is a linear combination of the restricted fields $\xi_{\alpha} \mid \mathcal{W}$ and, setting $g_{\beta}=\lambda_{\beta}^{\alpha} f_{\alpha}$, the equality $\eta_{\beta}\left|\mathcal{W}=\lambda_{\beta}^{\alpha} \xi_{\alpha}\right| \mathcal{W}$ holds.

(b) The condition $\left[f_{\alpha}, f_{\alpha^{\prime}}\right] \mid \mathcal{W}=0$ carries over to $\left[g_{\alpha}, g_{\alpha^{\prime}}\right] \mid \mathcal{W}=0$.

We observe that, unless all the Lagrange brackets vanish on $\mathcal{W}$, the vector fields $\xi_{\alpha}$ and $\eta_{\beta}$ are not necessarily tangent to $\mathcal{W}$, the notations $\xi_{\alpha} \mid \mathcal{W}$ and $\eta_{\beta} \mid \mathcal{W}$ simply indicating vector fields along the sub-manifold $\mathcal{W}$.

Lemma 7.8. Let $\xi$ and $\eta$ be two Lie vector fields with hamiltonians $f$ and $g$ respectively and let us assume that $f(H)=g(H)=[f, g](H)=0$, where $H \in G(M)$ is a given point. Then, $[\xi, \eta]_{H}$ is a linear combination of $\left\{\xi_{H}, \eta_{H}\right\}$ and, in coordinates, $[\xi, \eta]_{H}=(\partial g / \partial y)_{H} \xi_{H}-(\partial f / \partial y)_{H} \eta_{H}$.

We next prove a result that extends Lemma 7.3.

Lemma 7.9. Let $\xi_{\alpha}, 1 \leq \alpha \leq \ell$, be a family of linearly independent Lie vector fields, $f_{\alpha}$ the corresponding family of hamiltonians and $\mathcal{N}$ a regularly embedded sub-manifold of $G(M)$ contained in the variety $\mathcal{W}$ defined by the equations $\left\{f_{\alpha}=0\right\}$. We assume, furthermore, that $\mathcal{N}$ is an integral manifold of $\mathcal{P}$, that $\left[f_{\alpha}, f_{\beta}\right] \mid \mathcal{W}=0$ and that the linear subspaces $\Xi_{H} \subset T_{H} G(M)$ generated by the vectors $\left\{\xi_{\alpha}(H)\right\}$ are transversal to $\mathcal{N}$ at every point $H \in \mathcal{N}$ (i.e., $\Xi_{H} \cap T_{H} \mathcal{N}=0$ ). Then the union of all the $\ell$-dimensional flows $\exp \sum t^{\alpha} \xi_{\alpha}(H)$ initiating at points $H \in \mathcal{N}$ is, for small values of the parameters $\left(t^{\alpha}\right)$ (in a sense analogous to that of Lemma 7.3), a regularly embedded integral sub-manifold $\tilde{N}$ of $\mathcal{P}$ contained in $\mathcal{W}$, its dimension being equal to $\operatorname{dim} \mathcal{N}+\ell$.

Proof. The condition $\left[f_{\alpha}, f_{\beta}\right] \mid \mathcal{W}=0$ implies that the vector fields $\xi_{\alpha}$ are tangent to $\mathcal{W}$ and that each flow $\exp t^{\alpha} \xi_{\alpha}(H)$, initiating at a point $H \in \mathcal{W}$, is contained in $\mathcal{W}$ (Lemma 7.6 in the special case where $f_{\alpha}=g_{\alpha}$ ). The transversality condition implies that the union of all these flows initiating at 
points of $\mathcal{N}$ is, for small values of the parameters $t^{\alpha}$, a regularly embedded sub-manifold $\tilde{\mathcal{N}}$ of dimension equal to $\operatorname{dim} \mathcal{N}+\ell$.

Lemma 7.5 asserts, further, that the differential forms $\left\{d f_{\alpha} \wedge \omega\right\}$ are linearly independent at every point of $\mathcal{W}$ hence, a fortiori, the differentials $\left\{d f_{\alpha}\right\}$ are also linearly independent along $\mathcal{W}$. It follows that $\mathcal{W}$ is a regularly embedded sub-manifold of $G(M)$. By Lemma 7.8, the distribution $\Xi$, defined on $\mathcal{W}$ by the vector fields $\xi_{\alpha} \mid \mathcal{W}$, is integrable and of course the flow $\exp \sum t^{\alpha} \xi_{\alpha}(H)$ is, in a neighborhood of $H$, an open subset of the integral leaf of $\Xi$ containing the point $H$.

We next observe that each Lie vector field $\xi_{\alpha}$ is an infinitesimal automorphism of the system $\mathcal{P}$ and that its restriction $\xi_{\alpha} \mid \mathcal{W}$ is a characteristic vector field of $\Xi$. The local 1-parameter group generated by any linear combination, with constant coefficients, $\sum a^{\alpha} \xi_{\alpha}(H)$ transforms integral manifolds of $\mathcal{P}$ into integral manifolds and preserves the leaves of $\Xi$.

Finally, let $K \in \widetilde{\mathcal{N}}$ be an element of the form $K=\exp \sum t^{\alpha} \xi_{\alpha}(H)$, with $H \in \mathcal{N}$, and denote by $\phi_{t}$ the local 1-parameter group generated by the vector field $\sum a^{\alpha} \xi_{\alpha}(H)$. Then, since the transversality is preserved by diffeomorphisms, $T_{K} \widetilde{\mathcal{N}}=T \phi_{1}\left(T_{H} \mathcal{N}\right) \oplus T \phi_{1}\left(\Xi_{H}\right)$ and, on account of the above remarks, it follows that $\Omega$ vanishes on $T \phi_{1}\left(T_{H} \mathcal{N}\right)=T_{K} \phi_{1}(\mathcal{N})$ as well as on $T \phi_{1}\left(\Xi_{H}\right)=\Xi_{K}$, hence it also vanishes on $T_{K} \widetilde{\mathcal{N}}$, the sub-manifold $\widetilde{\mathcal{N}}$ being therefore an integral manifold of $\mathcal{P}$ contained in $\mathcal{W}$.

Remark 7.10. The existence of the integral manifold $\widetilde{\mathcal{N}}$ being only assured locally, we can relax the Lagrange bracket condition by requiring that $\left[f_{\alpha}, f_{\beta}\right] \mid \mathcal{W}$ vanishes only in a neighborhood of $\mathcal{N}$ and we can as well only require that the vector fields $\left\{\xi_{\alpha}\right\}$ be linearly independent along the sub-manifold $\mathcal{N}$. We could also compute directly the tangent space, at the point $K$, to the flow $\exp \sum t^{\alpha} \xi_{\alpha}(H)$ by using the formula of [5, Proposition 4.1]. However, Lemma 7.8 seems to be a more convenient technical device.

We finally need the following linear algebraic result:

Lemma 7.11. Let $\mu$ be an exterior 2-form of rank $2 n$ defined on a real or complex vector space $V, H$ a hyperplane of $V, \mu_{1}$ the induced form on $H$ and $A=\{v \in V \mid i(v) \mu=0\}$ the annihilator of $\mu$.

(a) If $\operatorname{dim} V=2 n$ then $\operatorname{rank} \mu_{1}=2 n-2$.

(b) If $\operatorname{dim} V=2 n+1$ and if $A \cap H=0$, then $\operatorname{rank} \mu_{1}=2 n$.

(c) If $\operatorname{dim} V=2 n+1$ and if $A \cap H \neq 0$, then $\operatorname{rank} \mu_{1}=2 n-2$.

Proof. (a). Since $\operatorname{dim} H=2 n-1$, the form $\mu_{1}$ has a non-trivial annihilator $A_{1}$. However, $v \in V \mapsto i(v) \mu \in V^{*}$ being an isomorphism, the kernel of 
the restricted map $v \in V \mapsto(i(v) \mu) \mid H \in H^{*}$ is 1-dimensional and therefore $\operatorname{dim} A_{1}=1$.

(b) and (c). Under the present hypotheses, $\operatorname{dim} A=1$. If $A \cap H=0$, then the annihilator $A_{1}$ is necessarily trivial otherwise any non-null vector $v \in A_{1}$ would also belong to $A$. If $A \cap H \neq 0$ or, equivalently, if $A \subset H$, then $A \subset A_{1}$ and, factoring all the data to the quotient space $V / A$, we recover the data of the first case.

Theorem 7.12. Let $\mathcal{S}$ be a system of regular first order partial differential equations of rank $q<n+1$, on the manifold $M$, and $\left\{f_{\alpha}=0\right\}$ a set of local equations of $\mathcal{S}$. Let us also denote by $\xi_{\alpha}$ the Lie vector field with hamiltonian $f_{\alpha}$ and assume that $\left[f_{\alpha}, f_{\beta}\right] \mid \mathcal{S}=0$ for all the indices $\alpha$ and $\beta$. Then the following statements hold.

(a) The vector fields $\left\{\xi_{\alpha}\right\}$ are tangent to $\mathcal{S}$ and the restrictions $\left\{\xi_{\alpha} \mid \mathcal{S}\right\}$ are everywhere linearly independent.

(b) Each $\xi_{\alpha} \mid \mathcal{S}$ is a characteristic vector field for the system $\mathcal{P} \mid \mathcal{S}$ and the family $\left\{\xi_{\alpha} \mid \mathcal{S}\right\}$ generates the characteristic distribution of $\mathcal{P} \mid \mathcal{S}$.

(c) Each q-dimensional flow $\exp \sum t^{\alpha} \xi_{\alpha}(H), H \in \mathcal{N}$, is locally (i.e., for small values of the parameters $t^{\alpha}$ ) independent of the particular choice of the local equations of $\mathcal{S}$, being therefore intrinsically associated to the system $\mathcal{S}$. Moreover, it is locally an open subset of the Cauchy characteristic containing the point $H$.

(d) Any maximal $(=n)$ dimensional integral manifold $\mathcal{N}$ of the Pfaffian system $\mathcal{P} \mid \mathcal{S}$ is the union of flows $\exp \sum t^{\alpha} \xi_{\alpha}(H)$ with $H \in \mathcal{N}$.

(e) Given an $(n-q)$-dimensional regularly embedded integral manifold $\mathcal{N}$ of $\mathcal{P} \mid \mathcal{S}$ transversal to the Cauchy characteristics, it extends to an $n$ dimensional integral manifold $\tilde{\mathcal{N}}$ by taking the union of all the flows $\exp \sum t^{\alpha} \xi_{\alpha}(H)$ initiating at points $H \in \mathcal{N}$.

Proof. Regularity condition $\Omega \mid \mathcal{S}_{H} \neq 0, H \in \mathcal{S}$, is obviously equivalent to the linear independence of the forms $\left\{\omega_{H},\left(d f_{\alpha}\right)_{H}\right\}$ and a simple calculation shows that the later property is equivalent, inasmuch, to the linear independence of the forms $\left\{\left(d f_{\alpha} \wedge \omega\right)_{H}\right\}$ hence, since $\mathcal{S}$ is regular, we infer from Lemma 7.5 that the vectors $\left\{\left(\xi_{\alpha}\right)_{H}\right\}$ are linearly independent along $\mathcal{S}$.

The tangency, to the manifold $\mathcal{S}$, of the vector fields $\left\{\xi_{\alpha}\right\}$ follows, by Lemma 7.6, from the bracket condition $\left[f_{\alpha}, f_{\beta}\right] \mid \mathcal{S}=0$. Observing that each $f_{\alpha}$ vanishes on $\mathcal{S}$, we also infer that $\left(\xi_{\alpha}\right)_{H} \in(\Sigma \mid \mathcal{S})_{H}$, for any $H \in \mathcal{S}$, hence the restrictions $\xi_{\alpha} \mid \mathcal{S}$ are characteristic vector fields of the system $\mathcal{P} \mid \mathcal{S}$. The remaining statements are now direct consequences of the Theorem 6.4 
for $\operatorname{dim} \mathcal{S}=2 n+1-q$ and therefore the dimension of the characteristic distribution of $\mathcal{P} \mid \mathcal{S}$ is equal to $(2 n+1-q)-(2 n-2 q+1)=q$.

It is often useful and always instructive to carry out a complete proof independently of the Theorem 6.4 and the argument runs as follows. Denote by $\Xi$ the distribution, on the manifold $\mathcal{S}$, generated by the vector fields $\xi_{\alpha} \mid \mathcal{S}$. Then $\Xi$ is intrinsically associated to the system $\mathcal{S}$ for, by Lemma 7.7, any other set of local equations for $\mathcal{S}$ provides the same distribution.

We also infer, from this Lemma, that the bracket condition is as well intrinsical. Next, we know from the proof of Lemma 7.9 that each flow $\exp \sum t^{\alpha} \xi_{\alpha}(H), H \in \mathcal{N}$ is, locally, an open subset of the integral leaf of $\Xi$ containing $H$ hence two such flows, associated to different sets of local equations for $\mathcal{S}$, will agree in a neighborhood of $H$, this settling part of the item (c).

Clearly, the item (e) is a restatement of Lemma 7.9. Since $\mathcal{P}$ and therefore $\mathcal{P} \mid \mathcal{S}$ admit integral manifolds of dimensions at most equal to $n$ and since a flow is the union of all the integral curves, initiating at $H$, of the characteristic vector fields $\sum a^{\alpha} \xi_{\alpha}$ (within the adequate bounds for the coefficients $a^{\alpha}$ ), the statement (d) is a direct consequence of Lemma 7.9, in the special case where $\ell=1$, for these characteristic fields must then be tangent to $\mathcal{N}$.

Finally, to prove that the flows $\exp \sum t^{\alpha} \xi_{\alpha}(H), H \in \mathcal{S}$, are locally open subsets of the Cauchy characteristics, it is enough to prove that the vector fields $\left\{\xi_{\alpha} \mid \mathcal{S}\right\}$ generate the characteristic distribution of $\mathcal{P} \mid \mathcal{S}$. For this, we consider the systems

$$
\mathcal{R}_{1}=\left\{f_{1}=0\right\}, \quad \mathcal{R}_{2}=\left\{f_{1}=f_{2}=0\right\}, \quad \ldots, \quad \mathcal{R}_{q}=\mathcal{R}=\left\{f_{\alpha}=0\right\},
$$

and argue as follows: The restriction $d \omega \mid \Sigma_{H}$ is a 2-form of rank $2 n$ defined on the vector space $\Sigma_{H}$ of dimension $2 n$ and $\left(\Sigma \mid R_{1}\right)_{H}$ is a hyperplane of $\Sigma_{H}\left(\Sigma \mid R_{1}\right.$ means the characteristic system of $\left.R_{1}\right)$. Therefore, according to Lemma 7.11, the rank of $d \omega \mid\left(\Sigma \mid R_{1}\right)_{H}$ is equal to $2 n-2$. Similarly, $\left(\Sigma \mid R_{2}\right)_{H}$ is a hyperplane in the $(2 n-1)$-dimensional vector space $\left(\Sigma \mid R_{1}\right)_{H}$ and, therefore, the rank of $d \omega \mid\left(\Sigma \mid R_{2}\right)_{H}$ is either $2 n-2$ or $2 n-4$.

Proceeding inductively, we infer that the rank of $d \omega \mid\left(\Sigma \mid R_{p}\right)_{H}$ is at least equal to $2 n-2 p$ hence, at the final stage, the rank of $d \omega \mid(\Sigma \mid R)_{H}$ is at least equal to $2 n-2 q$. It follows that the dimension of the characteristic subspace $\tilde{\Delta}_{H}$ of $\mathcal{P} \mid \mathcal{S}$ is, at most, equal to

$$
\operatorname{dim}(\Sigma \mid R)_{H}-(2 n-2 q)=(2 n-q)-(2 n-2 q)=q .
$$

Since $\Xi_{H} \subset \tilde{\Delta}_{H}$ and since $\operatorname{dim} \Xi_{H}=q$, we conclude that $\Xi=\tilde{\Delta}$.

The reader will have noticed that the present argument is nothing but a fancy way to deal with the Cartan argument introduced in the proof of the Theorem 6.4. 
Remark 7.13. We could of course enlarge the integral manifolds of $\mathcal{P} \mid \mathcal{S}$ by taking, step by step, either individual Lie vector fields $\xi_{\alpha}$ or sub-families $\left\{\xi_{\alpha_{i}}\right\}$ since such sub-families generate, in view of Lemma 7.8, integrable sub-distributions of $\Xi$.

When $q=1$, the above theorem reduces to the Theorem 7.4, the bracket condition then becoming trivial. When $q=n$, the $n$-dimensional distribution $\Sigma \mid \mathcal{S}$ is, under the hypotheses of the Theorem, generated by its characteristic vector fields and therefore is completely integrable.

We now examine the case $q=n+1$ excluded in the statement of the Theorem. Since the manifold $\mathcal{S}$ has, in this case, the dimension $n$, the system admits $n$-dimensional solutions containing any of its points if and only if $\Omega \mid \mathcal{S}=0$, the regularity hypothesis $\Omega \mid \mathcal{S} \neq 0$ being void of sense.

Lemma 7.14. Let $\mathcal{N}$ be an $n$-dimensional integral manifold of the system $\mathcal{P}$ and $\{f, g\}$ two functions vanishing on $\mathcal{N}$. Then $[f, g]$ also vanishes on $\mathcal{N}$.

In fact, the corresponding Lie vector fields $\xi$ and $\eta$ must be tangent to $\mathcal{N}$ otherwise we could enlarge this manifold to an $(n+1)$-dimensional integral manifold of $\mathcal{P}$. If, for example, $\xi_{H}$ were transverse to $\mathcal{N}$ then $(d f \wedge \omega)_{H} \neq 0$ hence $d f_{H} \neq 0$ and the level variety $f^{-1}(0)$ would become a manifold, in a neighborhood of $H$, containing $\mathcal{N}$. We could then, according to Lemma 7.3, enlarge the integral manifold $\mathcal{N}$. The tangency of $\xi$ and $\eta$ implies that of $[\xi, \eta]$ and therefore the hamiltonian $[f, g]=\langle[\xi, \eta], \omega\rangle$ vanishes on $\mathcal{N}$ since $\omega \mid \mathcal{N}=0$.

Lemma 7.15. Let $\mathcal{N}$ be an $n$-dimensional sub-manifold of $G(M)$ defined locally by the independent equations $\left\{f_{\alpha}=0\right\}$ and let us assume that $\left[f_{\alpha}, f_{\beta}\right] \mid \mathcal{N}=0$. Then $\Omega \mid \mathcal{N}=0$ and, furthermore, all the Lie vector fields $\xi_{\alpha}$ with hamiltonians $\left\{f_{\alpha}\right\}$, are tangent to $\mathcal{N}$, their restrictions generating $T \mathcal{N}$.

The proof will be omitted for being almost a repetition. The above Lemma shows that a system $\left\{f_{\alpha}\right\}$ of rank $n+1$ and satisfying the above bracket condition defines in fact an integral manifold of $\mathcal{P}$. The associated Pfaffian system $\mathcal{P} \mid \mathcal{N}$ is therefore null, its characteristics are regular and the characteristic distribution, equal to $T \mathcal{N}$, is generated by the vector fields $\xi_{\alpha} \mid \mathcal{N}$.

Corollary 7.16. In order that an $n$-dimensional sub-manifold $\mathcal{N}$ of $G(M)$ be an integral manifold of $\mathcal{P}$, it is necessary and sufficient that $\left[f_{\alpha}, f_{\beta}\right] \mid \mathcal{N}=0$ whatever the choice of the local equations $\left\{f_{\alpha}=0\right\}$ for $\mathcal{N}$. 
We can now settle the integrability problem for the system $\mathcal{S}$ namely, the existence of $n$-dimensional integral manifolds for the associated Pfaffian system $\mathcal{P} \mid \mathcal{S}$.

Theorem 7.17. A regular system $\mathcal{S}$ is integrable if and only if

$$
\left[f_{\alpha}, f_{\beta}\right] \mid \mathcal{S}=0
$$

whatever the choice of the local equations $\left\{f_{\alpha}=0\right\}$ for $\mathcal{S}$.

When $\operatorname{rank} \mathcal{S}=q=n+1$, the result is a restatement of the last Corollary. Assuming that $q \leq n$, the necessity of the condition follows from Lemma 7.14 and the sufficiency from the Theorem 7.12 for, under the stated condition of regularity, the Pfaffian system $\mathcal{P} \mid \mathcal{S}$ has regular characteristics with the appropriate dimension ( $c f$. the Corollary 6.5).

Remark 7.18. We infer, from Lemma 7.7, that the integrability condition, expressed in terms of the Lagrange bracket, is intrinsically associated to the equation $\mathcal{S}$. It could as well be restated as follows. The bracket of any two functions vanishing on $\mathcal{S}$ also vanishes on $\mathcal{S}$. Geometrically speaking, the condition states that, in a neighborhood of any point $H \in \mathcal{S}$, there exist $q$ Lie vector fields $\xi_{\alpha}$ with independent hamiltonians vanishing on $\mathcal{S}$ and such that $\left[\xi_{\alpha}, \xi_{\beta}\right]_{y} \in \Sigma_{y}$ whenever $y \in \mathcal{S}$. Since the Lagrangean bracket $[f, g]$ reduces, on the variety defined by the equations $f=g=0$, to the Jacobi bracket ( $c f$. , Part II, section 2), it follows that the above bracket condition boils down, when $q=n$, to the classical Frobenius integrability criterion.

We end up this section by discussing the infinitesimal contact automorphisms of the partial differential equations under consideration. The results will become useful in the second part.

Clearly, a Lie vector field $\xi$ is, in restriction to $\mathcal{S}$, an infinitesimal automorphism of the Pfaffian system $\mathcal{P} \mid \mathcal{S}$ if and only if it is tangent to $\mathcal{S}$. Such a vector field will be called an infinitesimal contact automorphism of the equation $\mathcal{S}$. Lemma 7.6 then specializes to the following result.

Lemma 7.19. A Lie vector field $\eta$ with hamiltonian $g$ is an infinitesimal contact automorphism of the equation $\mathcal{S}$ if and only if $\left[g, f_{\alpha}\right] \mid \mathcal{S}=0$ for any choice of local equations $\left\{f_{\alpha}=0\right\}$ for $\mathcal{S}$.

Given an element $H \in \mathcal{S}$, we denote by $\bar{g}_{H}$ the Lie algebra of all the germs of infinitesimal contact automorphisms of $\mathcal{S}$, at the point $H$, and set

$$
g_{H}=\bar{g}_{H} \mid \mathcal{S}, \quad h_{H}=\left\{g:\left[g, \bar{f}_{\alpha}\right] \mid \mathcal{S}=0\right\},
$$

where $g$ is a germ of a function at the point $H$ and $\left\{\bar{f}_{\alpha}=0\right\}$ indicates the germs, at $H$, of a set of local equations for $\mathcal{S}$ at $H$. 
Theorem 7.20. The Lie algebra $g_{H}$ is isomorphic to $h_{H} /\left(f_{\alpha}\right)^{2}$, where $\left(f_{\alpha}\right)$ denotes the ideal (in the sense of associative algebras) generated, in the algebra of all the germs, by the functions $f_{\alpha}$.

Proof. Let $\eta$ be an infinitesimal contact automorphism of $\mathcal{P} \mid \mathcal{S}$ with hamiltonian $g$ and let us assume that $\eta \mid \mathcal{S}=0$. Then $g \mid \mathcal{S}=0$ and, by writing locally $g=\sum \lambda^{\alpha} f_{\alpha}$, where $\left\{f_{\alpha}\right\}$ is a set of local equations for $\mathcal{S}$, we infer that $0=(d g \wedge \omega)_{H}=\sum \lambda^{\alpha}(H)\left(d f_{\alpha} \wedge \omega\right)_{H}$ whenever $H \in \mathcal{S}$, hence $\lambda^{\alpha} \mid \mathcal{S}=0$. Conversely, if $g \in\left(f_{\alpha}\right)^{2}$, then $\eta \mid \mathcal{S}=0$. It should be noted that the infinitesimal contact automorphisms of $\mathcal{P} \mid \mathcal{S}$ coincide with those of its characteristic system.

In all the preceding discussion, the scalar hamiltonians $f$ can always be replaced by $\Phi$-valued hamiltonians $h$ except, eventually, when expressions involving $d h$ and $h^{2}$ should occur. The differential $d h$ is meaningful and takes the role of $d f$ only at those points where $h$ vanishes. However, the expression $h^{2}$ is meaningless and the ideal $\left(f_{\alpha}\right)^{2}$ has to be replaced by the subset of all the germs of $\Phi$-valued sections $h$ vanishing to order 1 on $\mathcal{S}$.

Among the many Lie vector fields that we had the occasion to deal with in this section, let us recall those introduced in the very beginning and obtained, by prolongation, from vector fields coming from the base space $M$. In view of Lemma 7.19 and the local expression of such prolongations, the hamiltonians $f$ of such prolonged vector fields are characterized, locally, by the condition $\partial^{2} f / \partial p_{i} \partial p_{j}=0$ hence are semi-linear functions with respect to the variables $p_{i}$.

\section{REFERENCES}

[1] E. Cartan. Sur l'intégration de certains systèmes de Pfaff de caractère deux. Bull. Soc. Math. de France, 29:233-302, 1901.

[2] E. Cartan. Sur l'intégration des systèmes d'équations aux différentielles totales. Annales Sci. Ecole Norm. Sup., 18:241-311, 1901.

[3] E. Cartan. Sur les systèmes en involution d'équations aux dérivées partielles du second ordre à une fonction inconnue de trois variables indépendantes. Bull. Soc. Math. France, 39:352-443, 1911.

[4] E. Cartan. Sur l'équivalence absolue de certains systèmes d'équations différentielles et sur certaines familles de courbes. Bull. Soc. Math. de France, 42:12-48, 1914.

[5] A. Kumpera. Intégration des distributions singulières sur les variétés banachiques. Istit. Analisi Globale e Applic., Consiglio Nazionale delle Ricerche, 1:1-67, 1982.

[6] A. Kumpera, D. Spencer. Lie Equations, Vol.1: General Theory. Princeton University Press, 1972.

[7] M. Kuranishi. Lectures on involutive systems of partial differential equations. Universidade de São Paulo, 1967.

[8] S. Lie. Begründung einer Invariantentheorie der Berührungstransformationen. Math. Ann., 8:215-288, 1874. 
[9] S. Lie. Begründung einer Invariantentheorie der Berührungstransformationen. Math. Ann., 9:289-303, 1875.

[10] S. Lie. Zur Theorie der Berührungstransformationen. Leipz. Abh., 14:537-562, 1875.

[11] P. Olver. Applications of Lie groups to differential equations, volume 107. Springer Science and Business Media, 2000.

Received: October 5, 2018, accepted: February 3, 2019.

\author{
Antonio Kumpera \\ State University of Campinas \\ CAmpinas, SP, BRAZIL \\ Email: antoniokumpera@gmail.com \\ ORCID: orcid.org/0000-0001-8290-4254
}

